\title{
Visualization of flows of a viscous conductive liquid with the presence of impurities in the flow field corresponding to exact solutions of the MHD equations
}

\author{
V. A. Galkin ${ }^{1, A, B}$, A.O. Dubovik ${ }^{2, A, B}$ \\ A Surgut Branch of SRISA \\ B Surgut State University \\ ${ }^{1}$ ORCID: 0ooo-0002-9721-4026, val-gal@yandex.ru \\ 2 ORCID: 0000-0002-4158-9646, alldubovik@gmail.com
}

\begin{abstract}
$\underline{\text { Abstract }}$
A system of equations for the magnetic hydrodynamics (MHD), supplemented by the convection-diffusion equation describing the flow of a viscous conducting incompressible fluid taking into account the presence of impurities in it is considered in this paper. Modern scientific visualization software, such as ParaView, provide a wide range of possibilities for visualization and, therefore, a clearer presentation of the structure of such flows, especially in three-dimensional case. This opportunity allows to analyze, draw conclusions, note the features of the investigated fluid flows, and was actively used in the preparation of this publication.

The MHD model is an active field of research for many scientists, however, most of the works are devoted exclusively to numerical modeling, and very little attention has been paid to exact solutions for these problems. The article discusses classes of exact solutions of the MHD equations and the associated convection-diffusion equation. The exact solutions found can be used for verification of developed numerical algorithms for solving the discussed problems. Moreover, the exact solutions found describe fluid flows in a porous medium and are related to the development of a core simulator that will allow to simulate intraplast effects. It is associated with the creation of a domestic technology "digital field" and the tasks of controlling the parameters of incompressible liquids. For the oil and gas industry, a solution of this class of task will allow to perform optimization effects of various types (mechanical, electromagnetic, thermal, etc.) in order to increase the oil recovery factor.

Keywords: magnetohydrodynamics, exact solutions, fluid flow visualization, convection-diffusion.
\end{abstract}

\section{Introduction}

The system of equations of magnetohydrodynamics (MHD) is considered [1, 2], describing the flow of a viscous conducting fluid in the region, taking into account the presence impurities $\left\{x_{1}, x_{2}, x_{3}\right\} \in D, t>0$ :

$$
\begin{gathered}
\frac{\partial \mathbf{H}}{\partial t}=\operatorname{curl}[\mathbf{u} \times \mathbf{H}]+\mu_{m} \Delta \mathbf{H}, \\
\operatorname{div} \mathbf{u}=0, \\
\frac{\partial \mathbf{u}}{\partial t}+(\mathbf{u} \cdot \nabla) \mathbf{u}=-\frac{1}{\rho_{0}} \nabla\left(p+\frac{\mathbf{H}^{2}}{8 \pi}\right)+\frac{1}{4 \pi \rho_{0}}(\mathbf{H} \cdot \nabla) \mathbf{H}+\mu \Delta \mathbf{u}, \\
\operatorname{div} \mathbf{H}=0,
\end{gathered}
$$




$$
\frac{\partial n}{\partial t}+(\mathbf{u} \cdot \nabla) n=\chi \Delta n,
$$

where $\mathbf{H}$ is the magnetic field strength vector, $\mathbf{u}$ is the fluid velocity vector, $t$ is the time, $\mu_{m}$ is the magnetic viscosity of the liquid, $\rho_{0}$ is the fluid density, $p$ is the pressure, $\mu$ is the kinematic viscosity, $n$ is the concentration of impurities in the liquid, $\chi$ is the coefficient diffusion. Note that the pressure field $p$ is determined from equations (1) - (5) accurate to arbitrary function of time.

The classes of flows considered in the work have a complex structure, especially flows in space, therefore, for a clearer presentation and analysis of them, it is necessary to use modern means of scientific visualization. ParaView (https://www.paraview.org) was used in the preparation of this post.

The creation of the domestic technology "digital field" [3-5] involves the ability to develop a core simulator that allows you to simulate dynamics liquids depending on external influences (thermal, electromagnetic, chemical, mechanical, etc.) and to pose problems of their optimization in order to extracting profitability from the exploitation of deposits in the future. Impact research thermal effects on the flow structure are discussed in [6-9], including such viscous liquids like oil [8]. The presented class of tasks allows you to include hydrogeodynamic problems described in [10] in consideration of the reservoir deformation model .

The investigated model of the fluid flow taking into account convective diffusion is an intense field of research [11-14]. However, all these studies are devoted exclusively to the issues of the numerical solution of these problems. Numerical simulation results must be verified by exact solutions. This work is devoted to the representation of various classes of exact solutions of the MHD equations taking into account the presence of impurities in the flow field.

Consideration below the presented exact solutions is related to the issue of building a core simulator and describing the dynamics of a fluid in a porous medium. The simplest model of a porous medium comprises a discrete set of points - the grid nodes $\partial D$. Then the area of fluid flow is $D \equiv \mathbb{R}^{n} \backslash \partial D$. In this case, on the boundary of the flow region, adhesion condition. The exact solutions presented below are considered for different initial and boundary conditions depending on the specific submodels of model (1) - (5). In the exact solutions given below, set $\rho_{0}=\chi=1, \mu_{m}=\mu$. Solutions 2.1.2 and 3.2.2 have already been published in [15], the rest of the obtained expressions are published for the first time.

General comments to the figures presented in the article are as follows. White lines and arrows in the figure correspond to streamlines and the direction of the vector field, as fluid velocity vector rule. In paragraphs 2,3 , the figures correspond to the fragment flow areaa square with a side of $2 \pi$ centered at the origin, i.e., corners of the square correspond to points with all coordinates $\pm \pi$. In paragraphs 4,5 , the figures illustrate the flows on a sphere of radius 5. As a rule, unless otherwise specified, animation on in the figures corresponds to the change in the flow in time from $t=0$ to $t=1$ with a step of 0.2 .

\section{Equations of hydrodynamics}

The following submodel of equations (1) - (5) is considered, which consists of the equation (2) and the Navier-Stokes equations:

$$
\frac{\partial \mathbf{u}}{\partial t}+(\mathbf{u} \cdot \nabla) \mathbf{u}=-\frac{1}{\rho_{0}} \nabla p+\mu \Delta \mathbf{u},
$$

At the boundary of the flow region, the adhesion conditions are satisfied

$$
\left.\mathbf{u}\right|_{\partial D}=0 .
$$


All examples of exact solutions given in paragraphs 2, 3 are considered in the area having a periodic structure and consisting of squares or cubes of length $2 \pi$. In if the specified structures have a different size, then as a result of using the replacement of space-time variables, arrive at the formulas below.

\subsection{Exact solutions in the plane}

Let the domain $D$ be a flat mesh consisting of cells with a length and width equal to $2 \pi$.

1. If the initial condition has the form

$$
\left.\mathbf{u}\right|_{t=0}=\left\{\cos x_{2} ;-\cos x_{1} ; 0\right\}
$$

then the exact solution to problem (2), (6), (7) has the form

$$
\mathbf{u}=e^{-\mu t}\left\{\cos x_{2} ;-\cos x_{1} ; 0\right\}, p=-e^{-2 \mu t} \sin x_{1} \sin x_{2} \text {. }
$$

In this case, the boundary of the flow region $\partial D$ are points $x_{1}, x_{2}=\frac{\pi}{2}+\pi n, n \in \mathbb{Z}$. It can be seen from fig. 1, that the fluid flow is structured into squares, inside which vortices with centers located at grid nodes $\partial D$ rotate, in which the flow velocity vanishes. At the borders of squares located at corners $\pm \frac{\pi}{4}$ and centers on mesh, the fluid slides along a tangent. A similar flow structure for a conducting liquid was observed when describing the exact solution of the MHD equations with the condition sliding on the parallelepiped boundary [16]. Note that the flows studied here are known in nature as square waves. These waves are a grid pattern of many squares on the surface seas. These waves are strong enough and can change the trajectory of ships in unpredictable direction. Square waves are formed from two different groups of waves converging at different angles, not exceeding, as a rule, $45^{\circ}$.

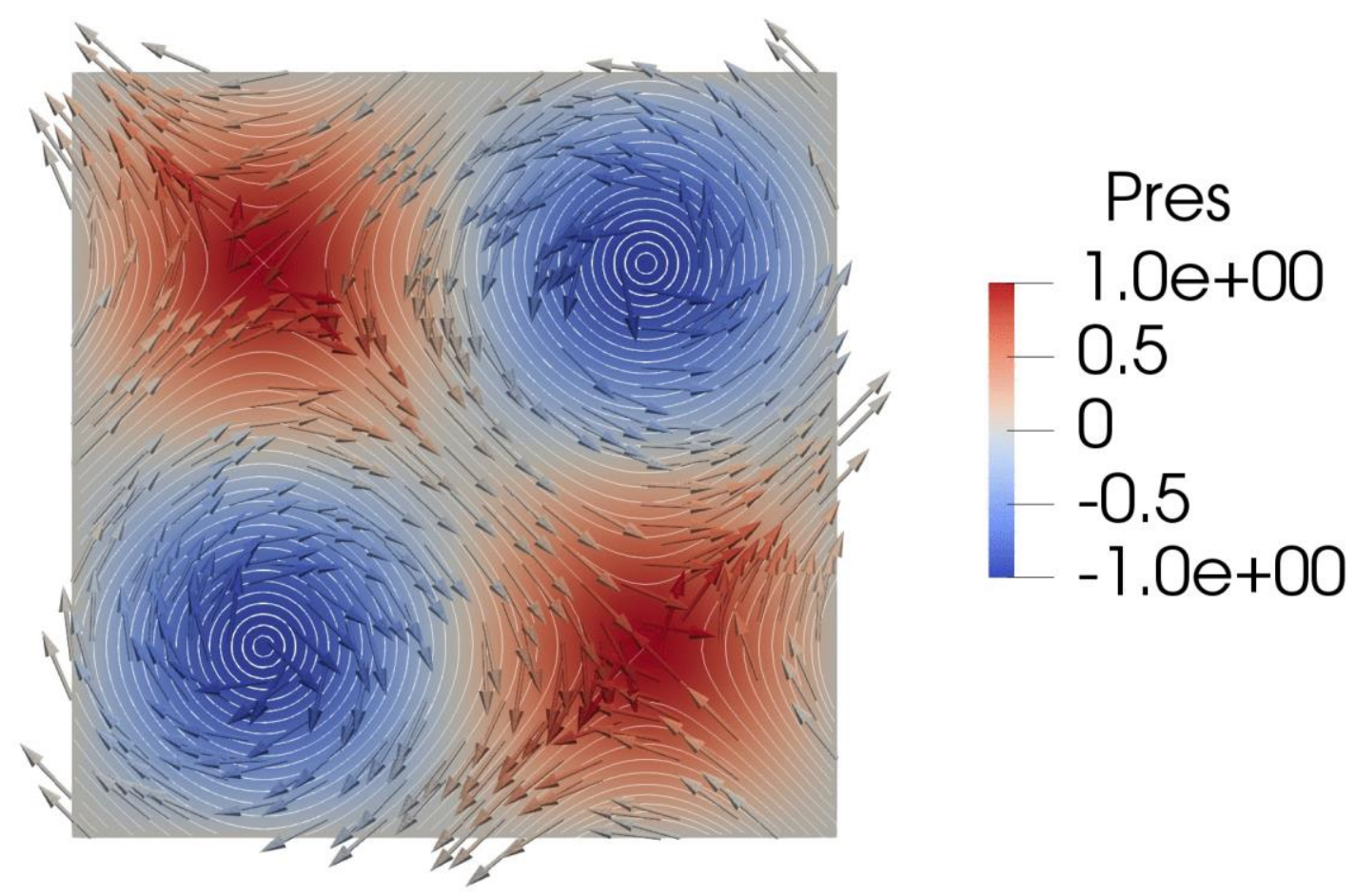

Fig. 1. Flow structure corresponding to solution 2.1.1

2. If the initial condition has the form

$$
\left.\mathbf{u}\right|_{t=0}=\left\{\sin \left(\sqrt{\lambda} x_{2}\right) ; \sin \left(\sqrt{\lambda} x_{1}\right) ; 0\right\}, \lambda>0,
$$

then the exact solution to problem (6) - (8) has the form 


$$
\mathbf{u}=e^{-\mu \lambda t}\left\{\sin \left(\sqrt{\lambda} x_{2}\right) ; \sin \left(\sqrt{\lambda} x_{1}\right) ; 0\right\}, p=e^{-2 \mu \lambda t} \cos \left(\sqrt{\lambda} x_{1}\right) \cos \left(\sqrt{\lambda} x_{2}\right) .
$$

In this case, the boundary of the flow region $\partial D$ are the points $x_{1}, x_{2}=\frac{\pi n}{\sqrt{\lambda}}, n \in \mathbb{Z}$. Fig. 2 shows the flow structure corresponding to the considered in this point to the exact solution at $\lambda=4$. In the same way as in the previous case, the fluid flow is divided into squares with rotating vortices in the center of which the flow velocity is zero.

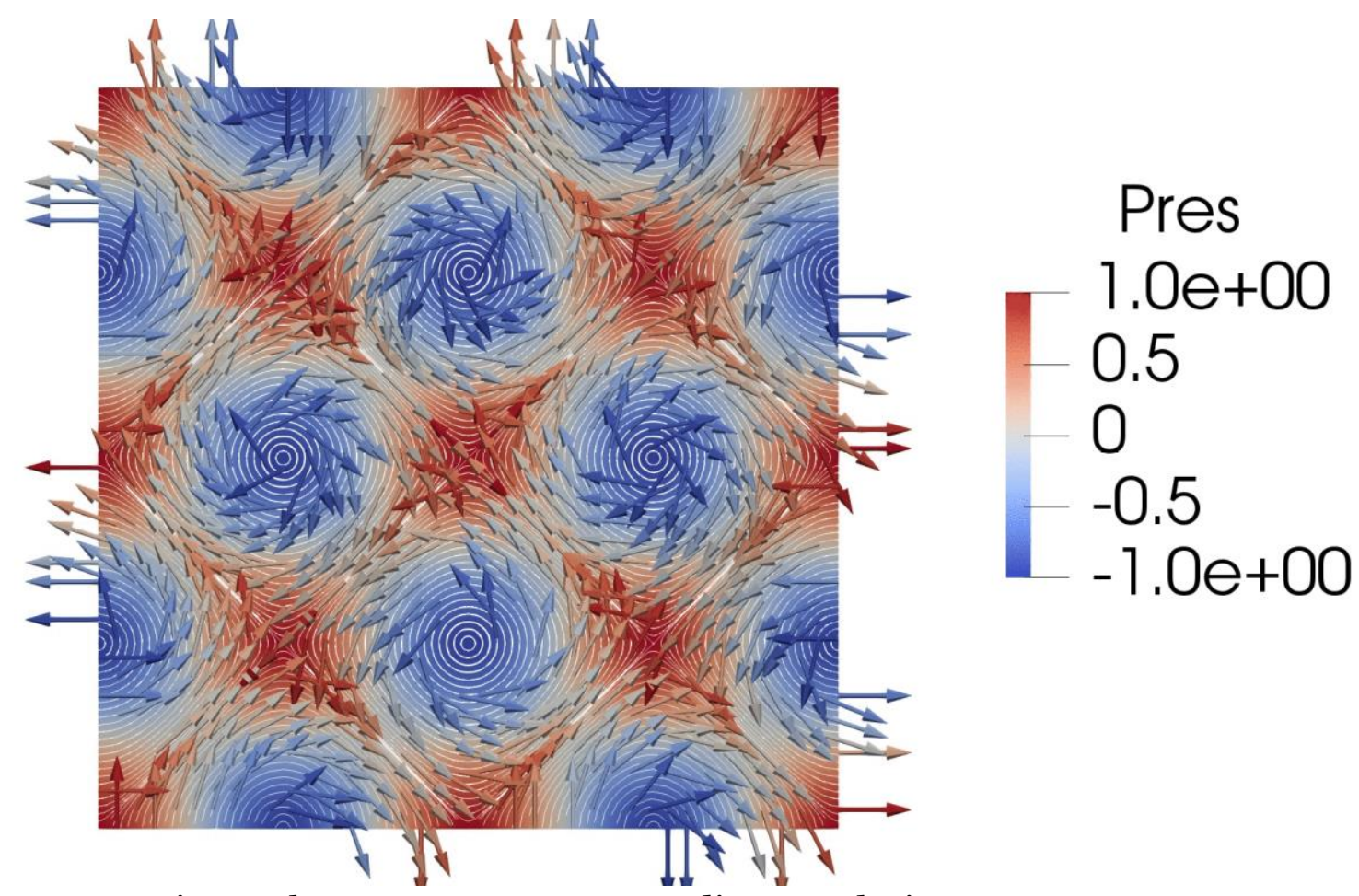

Fig. 2. Flow structure corresponding to solution 2.1.2

\subsection{Exact solutions in space}

1. If the initial condition has the form

$$
\left.\mathbf{u}\right|_{t=0}=\left\{\exp \left(\lambda\left(x_{3}-\varphi(0)\right)\right) \sin x_{2} ; \exp \left(-\lambda\left(x_{3}-\varphi(0)\right)\right) \sin x_{1} ; \varphi^{\prime}(0)\right\}
$$

where $\lambda$ is an arbitrary parameter, then the exact solution to problem (2), (6), (7) has the form

$$
\begin{gathered}
\mathbf{u}=\left\{\exp \left(\left(\lambda^{2}-1\right) \mu t\right) \exp \left(\lambda\left(x_{3}-\varphi(t)\right)\right) \sin x_{2} ; \exp \left(\left(\lambda^{2}-1\right) \mu t\right) \exp \left(-\lambda\left(x_{3}-\varphi(t)\right)\right) \sin x_{1} ; \varphi^{\prime}(t)\right\}, \\
p=\exp \left(2\left(\lambda^{2}-1\right) \mu t\right) \cos x_{1} \cos x_{2}-\varphi^{\prime \prime}(t) x_{3},
\end{gathered}
$$

where $\varphi(t)$ is an arbitrary function of time. In this case, the boundary of the flow region $\partial D$ are the points $x_{1}, x_{2}=\pi n, n \in \mathbb{Z}$.

The change in the flow structure over time is illustrated, corresponding to the considered exact solution, depending on time at parameter $\lambda=2$ and at $\varphi(t)=\frac{t^{2}}{2}$ in fig. 3-5. The figures illustrate the flow of liquid in plane $x_{1} O x_{2}$ and planes parallel to it, passing through points with coordinates $(0,0,1)$ for Fig. 4 and $(0,0,2)$ for Fig. 5 . It follows from the illustrations that the flow fluid becomes less structured over time, and with increasing values of the coordinate $x_{3}$, the flow is straightened along the axis $O x_{1}$. Similar the remark is also true when decreasing the values $x_{3}$, however, straightening flow now occurs along the $O x_{2}$ axis. The pressure field acquires over time cellular structure. 


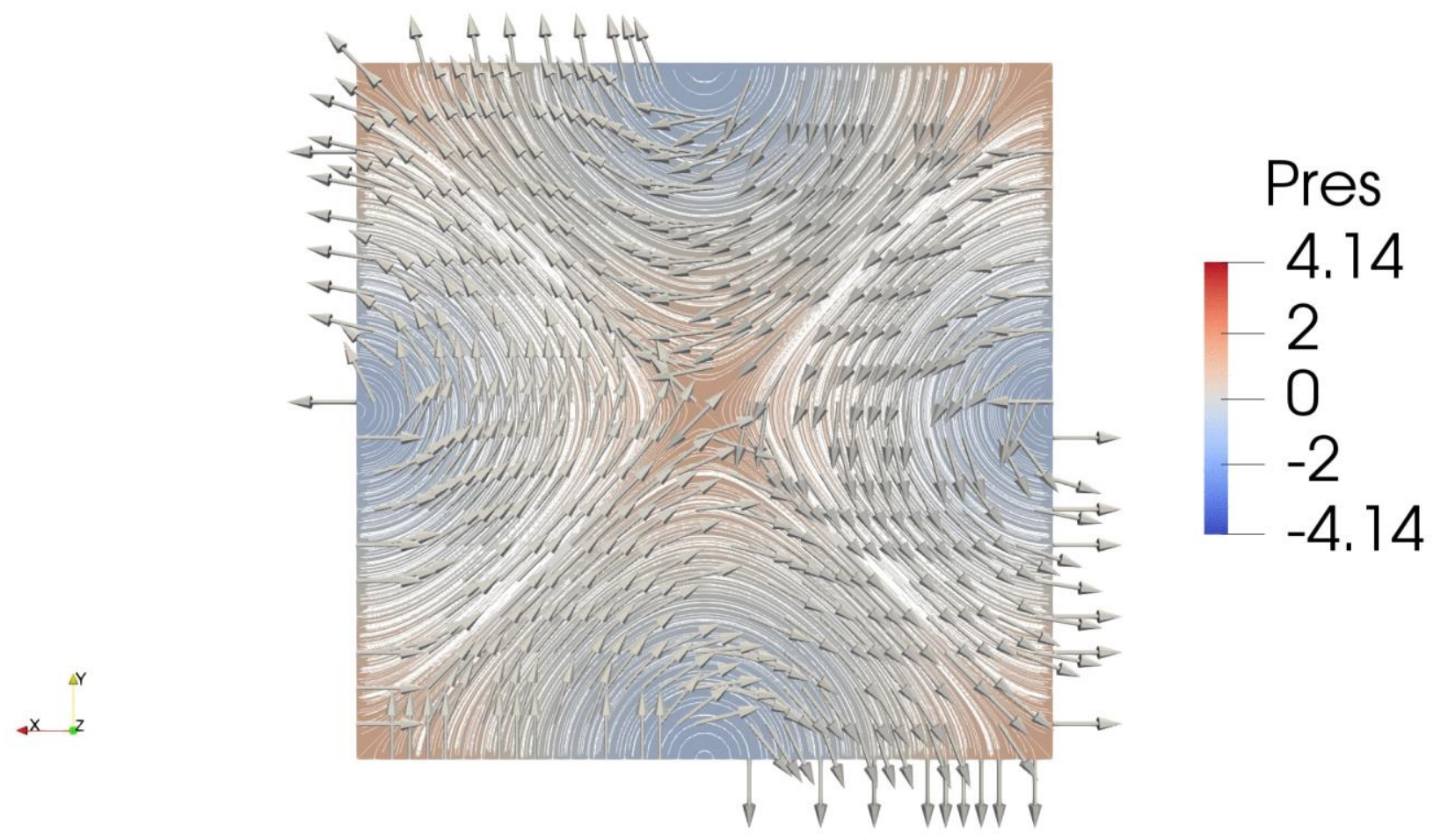

Fig. 3. The structure of the flow 2.2.1 in the plane $x_{1} O x_{2}$
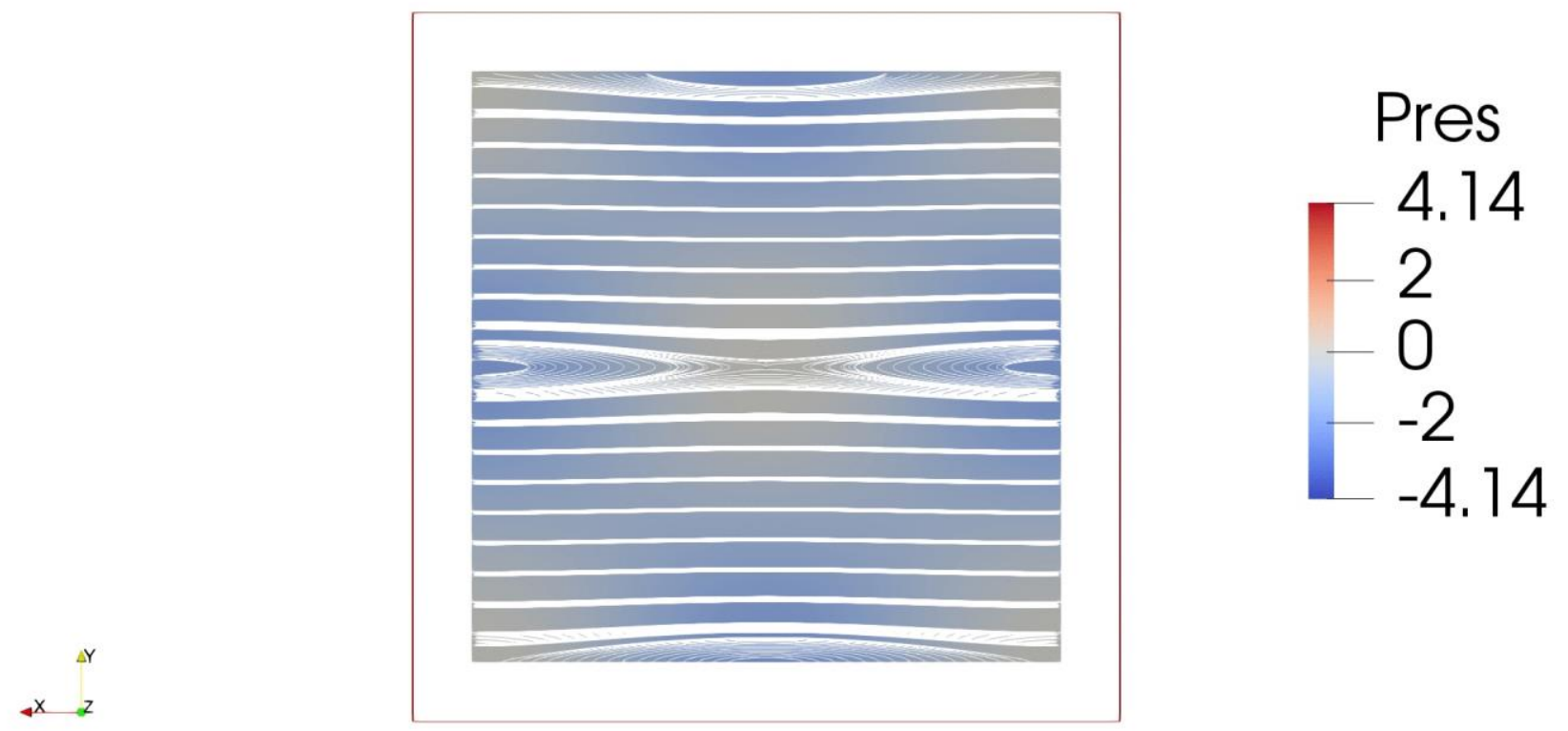

Fig. 4. The structure of the flow 2.2.1 in the plane parallel to $x_{1} O x_{2}$, passing through the point $(0,0,1)$ 

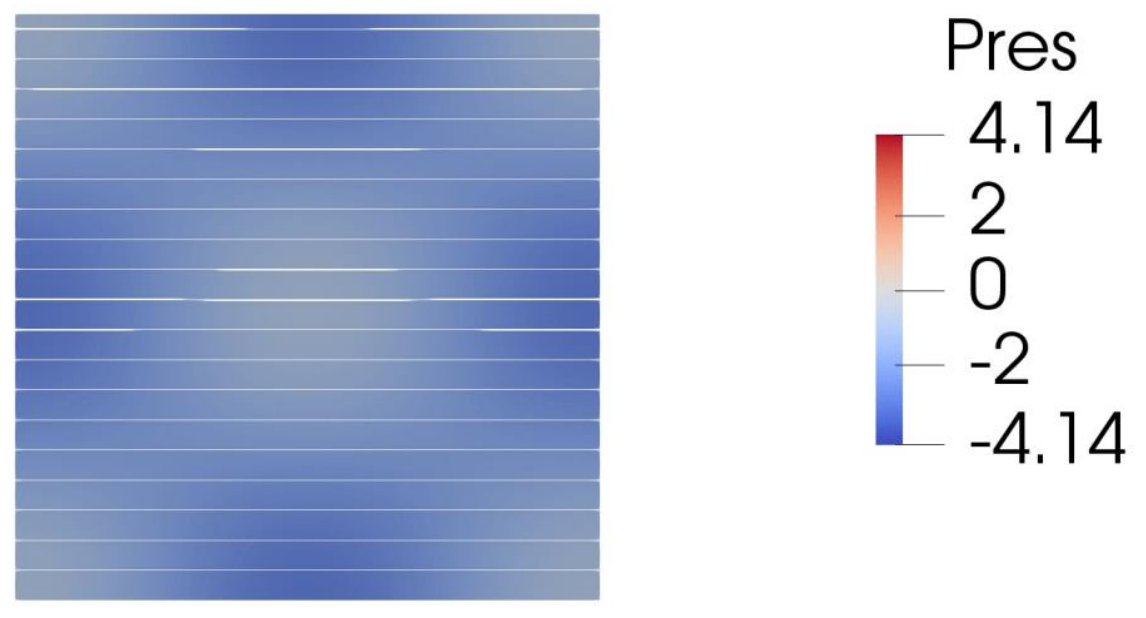

Fig. 5. The structure of the flow 2.2.1 in the plane parallel to $x_{1} O x_{2}$, passing through the point $(0,0,2)$

2. Let the domain $D$ be a spatial grid consisting of cells with length, width and height equal to $2 \pi$. If the initial condition is

$$
\left.\mathbf{u}\right|_{t=0}=\left\{\sin x_{2}-\cos x_{3} ; \sin x_{3}-\cos x_{1} ; \sin x_{1}-\cos x_{2}\right\},
$$

then the exact solution to problem (6) - (8) has the form

$$
\mathbf{u}=e^{-\mu t}\left\{\sin x_{2}-\cos x_{3} ; \sin x_{3}-\cos x_{1} ; \sin x_{1}-\cos x_{2}\right\}, p=-\frac{\mathbf{u}^{2}}{2} \text {. }
$$

In this case, the boundary of the flow region $\partial D$ are points $x_{1}=x_{2}=x_{3}=\frac{\pi}{4}+\pi n, n \in \mathbb{Z}$.

To make the idea of the structure of the fluid flow (Fig. 6-9) clearer it is necessary to perform the following transformation of coordinates: express all cosines, appearing in the exact solution for the velocity field through the sines and make the substitution

$$
\xi_{i}=\frac{x_{j}+x_{k}}{2}
$$

where all indices $i, j, k=1,2,3$ are different. The flow structure is illustrated in plane parallel to $x_{1} O x_{2}$ and passing through points of the form $x_{1}=x_{2}=x_{3}=-\pi,-\pi / 2,-\pi / 4, \pi / 4$ accordingly, depending on the time From Figures 6-9 it follows that the fluid flow has a structure to each cell, while the streamlines in the cells coincide in each case, but the direction of the flow in adjacent cells is reversed. Note, that when considering the flow in the plane parallel $x_{1} O x_{2}$ and passing through the points $x_{1}=x_{2}=x_{3}=\pi$ the same flow pattern is obtained as in Fig. 6, and in the case of the point $x_{1}=x_{2}=x_{3}=\pi / 2$ - the same flow pattern as in Fig. 7 . 


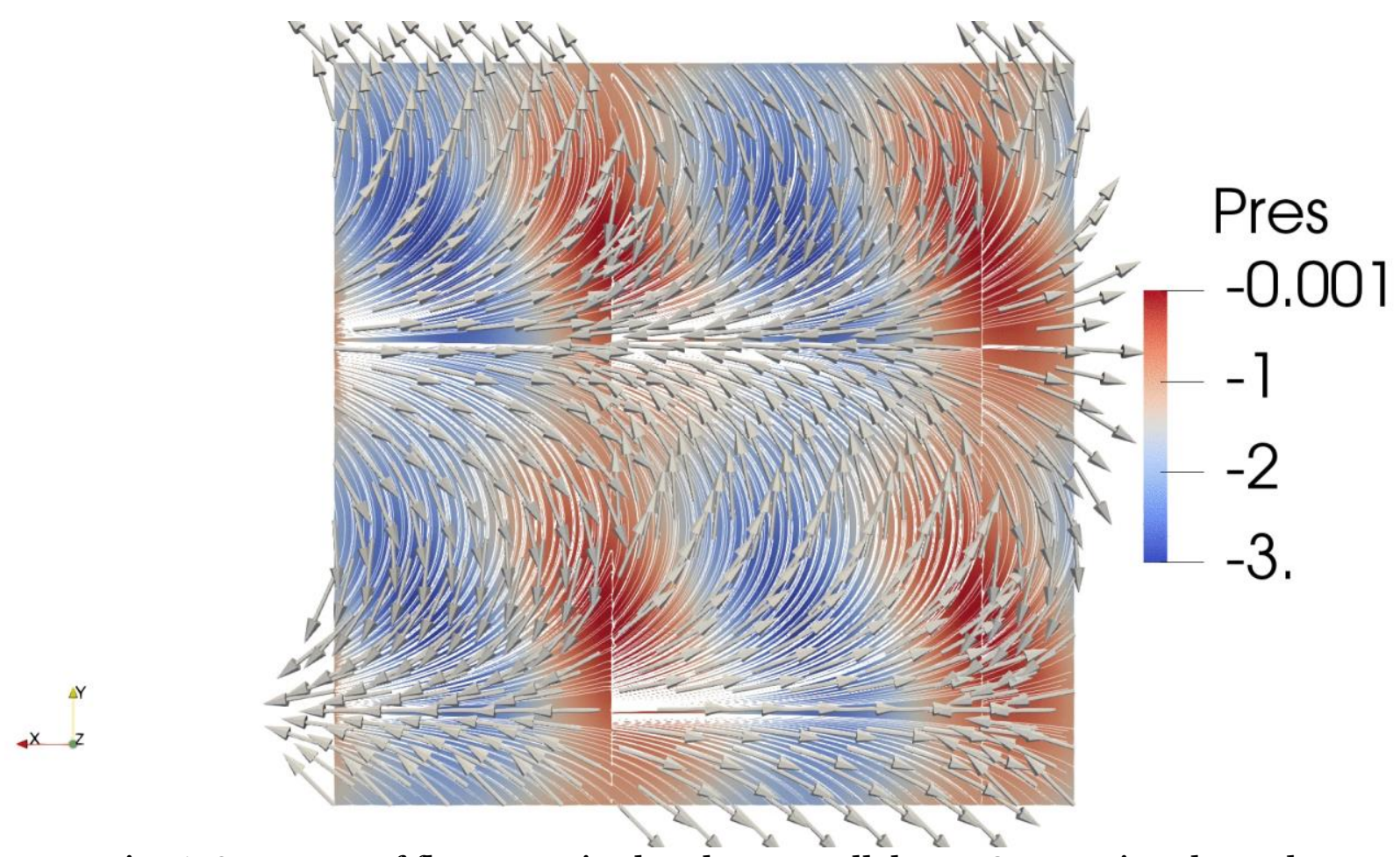

Fig. 6. Structure of flow 2.2.2 in the plane parallel to $x_{1} O x_{2}$ passing through the point $(-\pi,-\pi,-\pi)$

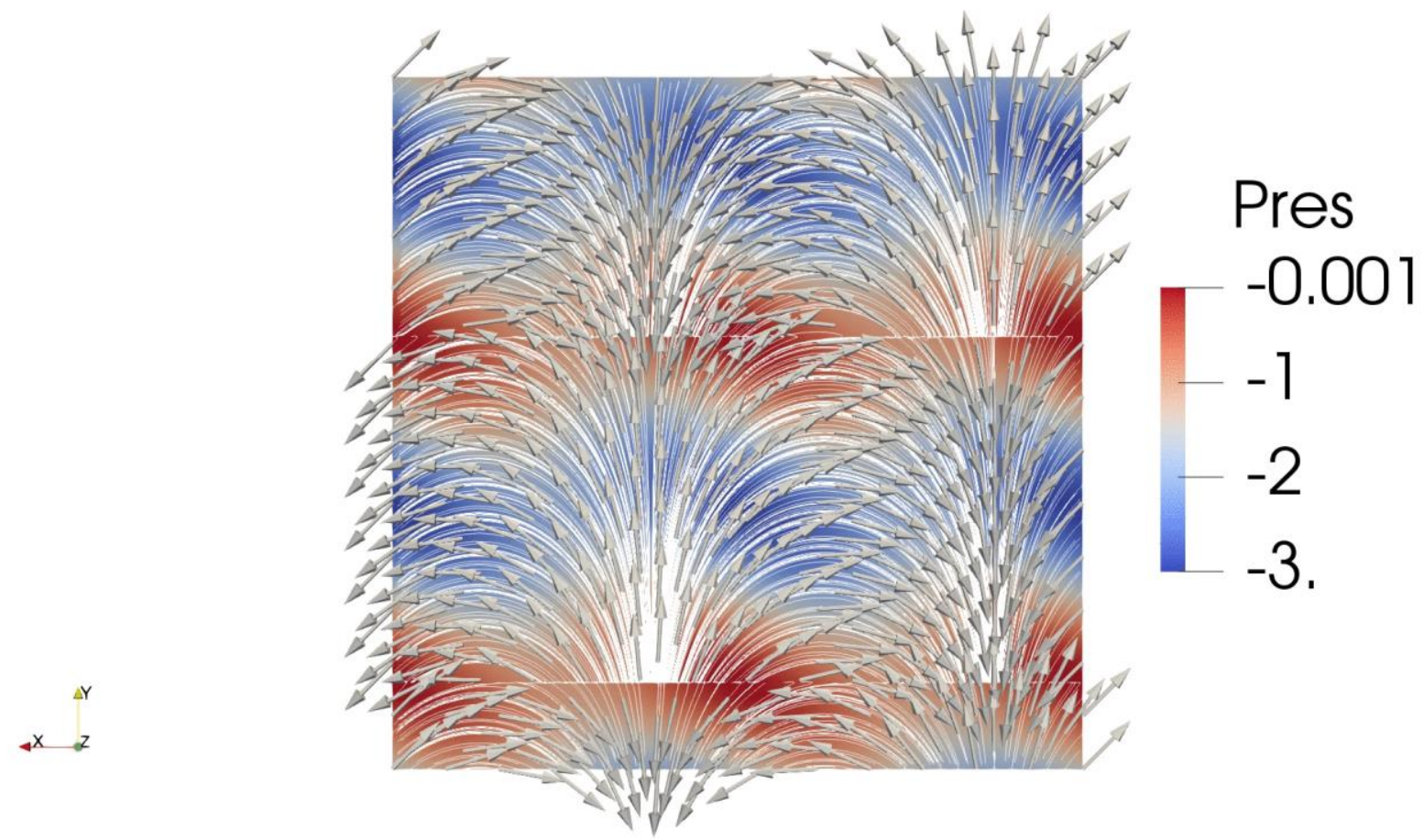

Fig. 7. The structure of the flow 2.2.2 in the plane parallel to $x_{1} O x_{2}$ passing through the point $(-\pi / 2,-\pi / 2,-\pi / 2)$ 


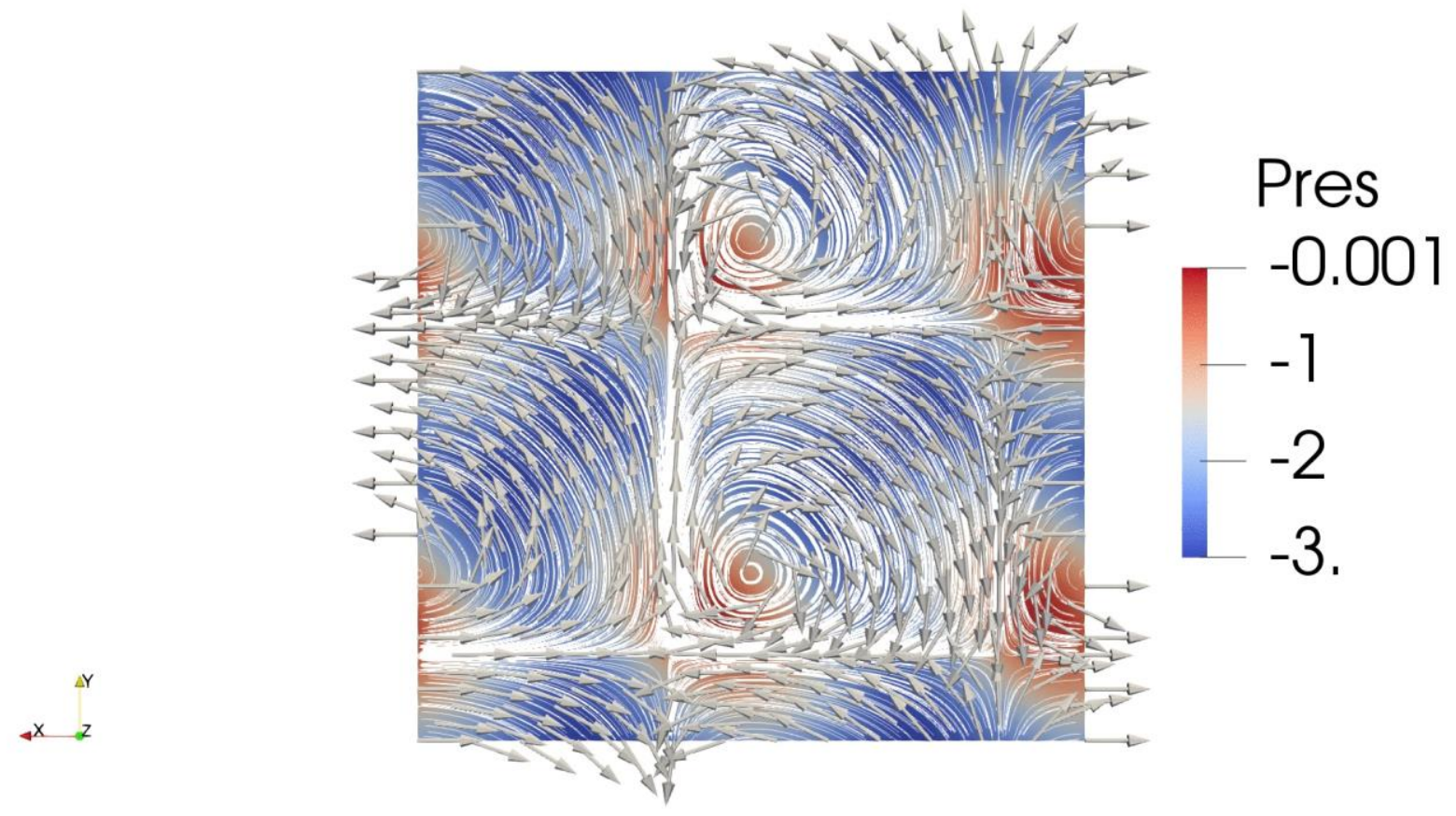

Fig. 8. Structure of the flow 2.2.2 in the plane parallel to $x_{1} O x_{2}$ passing through the point $(-\pi / 4,-\pi / 4,-\pi / 4)$

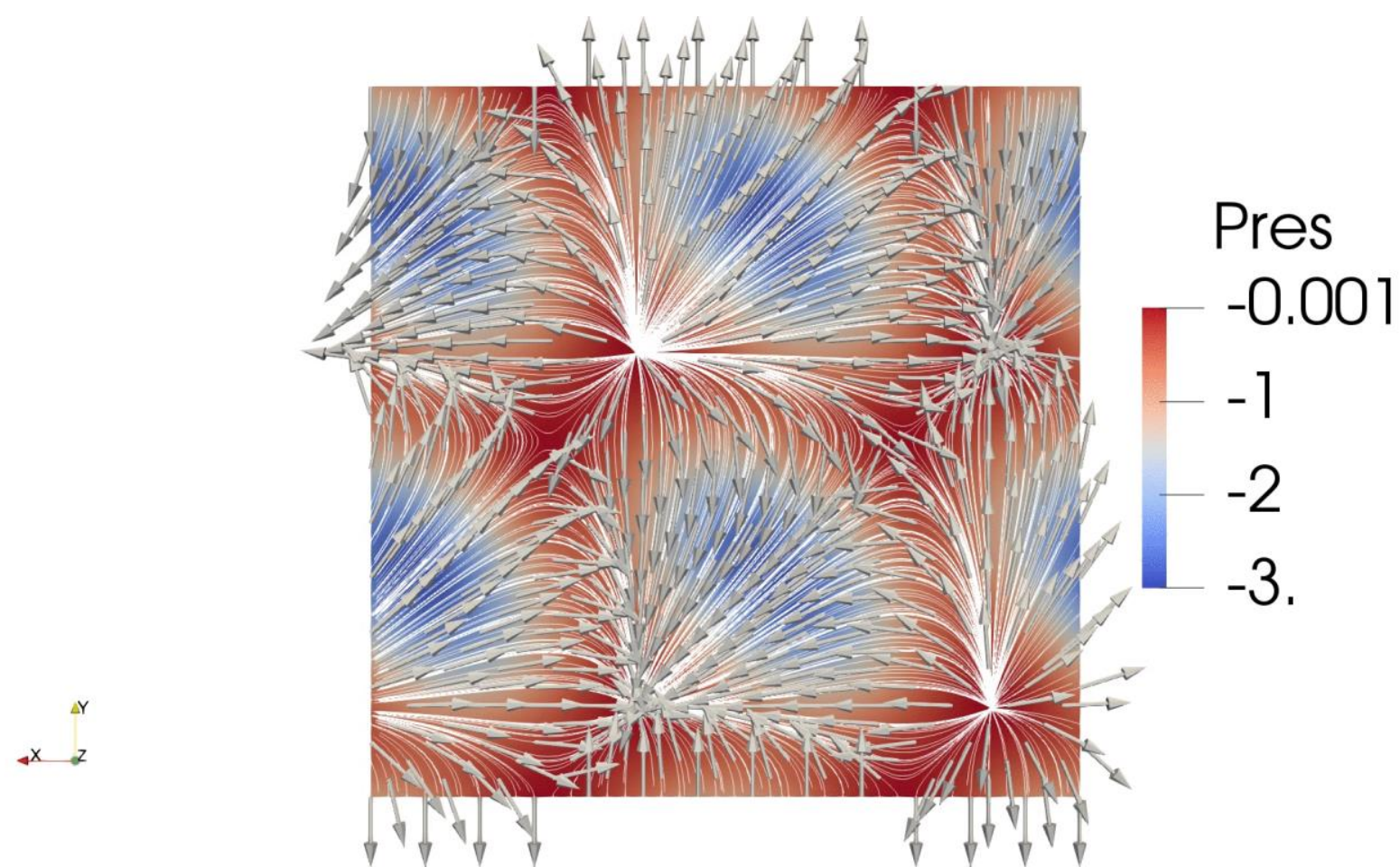

Fig. 9. The structure of the flow 2.2.2 in the plane parallel to $x_{1} O x_{2}$ passing through the point $(\pi / 4, \pi / 4, \pi / 4)$

\section{Equations of hydrodynamics with impurities}

The following submodel of equations (1) - (5) is considered, which consists of the equations (2), (5), (6). At the boundary of the flow region, conditions (7) are satisfied and

$$
\left.\frac{\partial n}{\partial \mathbf{N}}\right|_{\partial D}=0
$$

where $\mathbf{N}$ - normal vector to the boundary $\partial D$. 
Let also, as in the previous section, the domain $D$ have a periodic structure and consists of cubes with an edge of length $2 \pi$. If the initial condition is

$$
\begin{aligned}
& \left.\mathbf{u}\right|_{t=0}=\left\{\exp \left(\varepsilon x_{3}\right) \sin \lambda x_{2} ; \exp \left(-\varepsilon x_{3}\right) \sin \lambda x_{1} ; 0\right\}, \\
& \left.n\right|_{t=0}=\left\{\exp \left(-\varepsilon x_{3}\right) \cos \lambda x_{1}-\exp \left(-\varepsilon x_{3}\right) \cos \lambda x_{2}\right\},
\end{aligned}
$$

where $\lambda \neq 0$, then the exact solution to problem (2), (5) - (8) has the form

$$
\begin{gathered}
\mathbf{u}=\exp \left(\left(\varepsilon^{2}-\lambda^{2}\right) \mu t\right)\left\{\exp \left(\varepsilon x_{3}\right) \sin \lambda x_{2} ; \exp \left(-\varepsilon x_{3}\right) \sin \lambda x_{1} ; 0\right\}, \\
p=\exp \left(2 \mu t\left(\varepsilon^{2}-\lambda^{2}\right)\right) \cos \left(\lambda x_{1}\right) \cos \left(\lambda x_{2}\right), \\
n=\exp \left(\chi t\left(\varepsilon^{2}-\lambda^{2}\right)\right)\left(\exp \left(-\varepsilon x_{3}\right) \cos \left(\lambda x_{1}\right)-\exp \left(\varepsilon x_{3}\right) \cos \left(\lambda x_{2}\right)\right) .
\end{gathered}
$$

Moreover, everywhere in the domain $D$ the condition $(\mathbf{u} \cdot \nabla) n \equiv 0$, and the solution to problem (2), (5) - (8) is two-parameter and depends on the parameters $\varepsilon$ and $\lambda$. In this case, the border the flow domains $\partial D$ are straight lines parallel to the $O x_{3}$ axis and projecting into the points plane $x_{1} O x_{2}$ with coordinates $x_{1}, x_{2}=\frac{\pi n}{\lambda}, n \in \mathbb{Z}$.

For $\lambda=\mu$ obtain a stationary flow. Let $\lambda=\mu=2$, then the field is a flow, corresponding to a given exact solution, in planes parallel to $x_{1} O x_{2}$ and passing with the interval $\pi / 4$ through the points lying on the $O x_{3}$ axis starting from the point having coordinate $x_{3}=-\pi / 2$ and to the point with coordinate $x_{3}=\pi / 2$ is shown in Fig. 10, 11. Fig. 10 illustrates the concentration field, and Fig. 11 the pressure field. In fig. 12 the pressure field is illustrated in the section of the plane parallel to $x_{2} O x_{3}$. Similarly to the previous two figures here the plane passes through the points lying on the axis $O x_{1}$ and having coordinates of the form $(-\pi / 2+\pi n / 4,0,0), n=0,1, \ldots, 4$. From Fig. 10 it is seen that the impurity is located along streamlines, while streamlines significantly depend on the coordinate $x_{3}$. Fig. 11, 12 demonstrates the presence of a fixed structure in the pressure field.

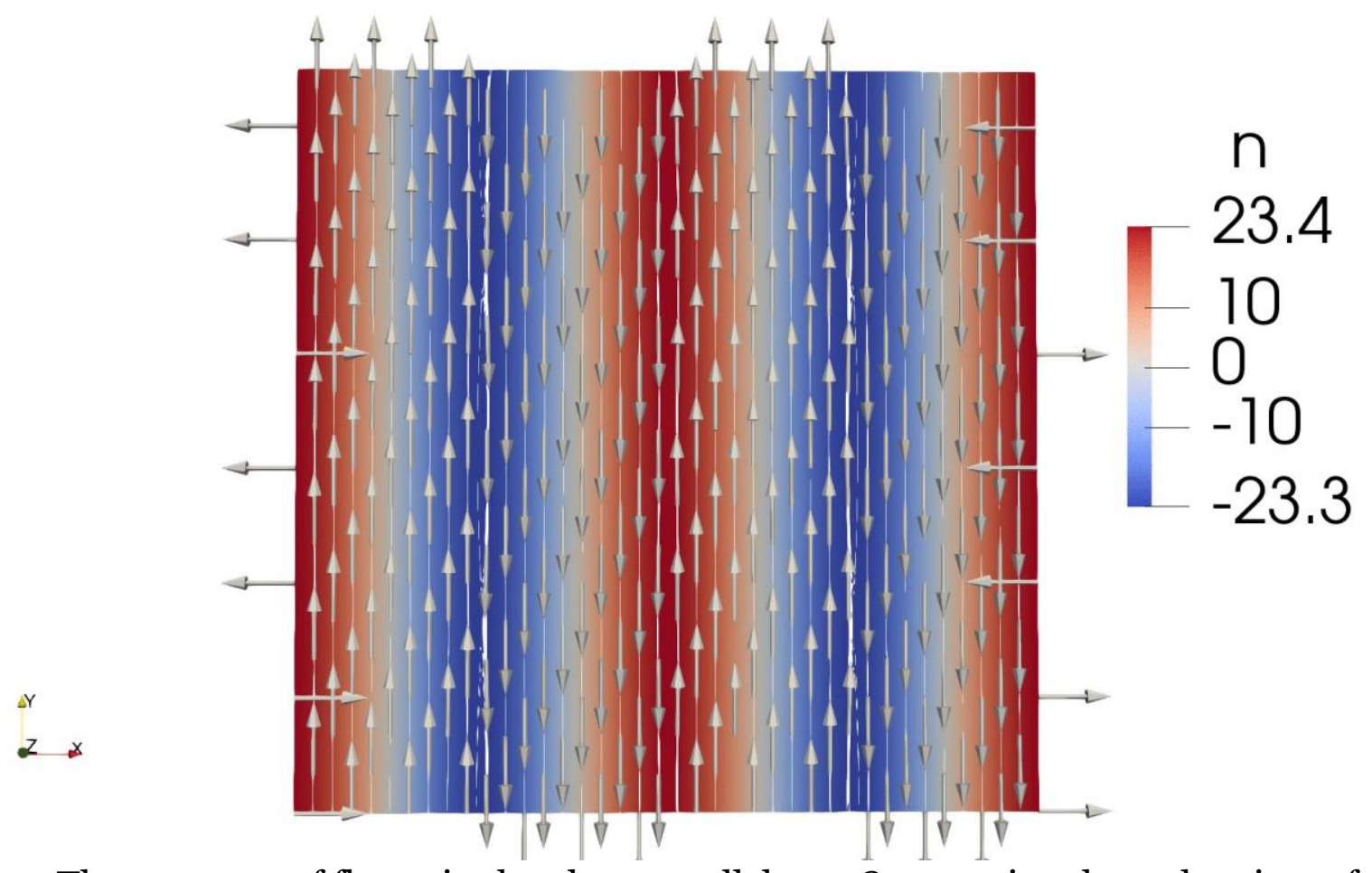

Fig. 10. The structure of flow 3 in the plane parallel to $x_{1} O x_{2}$, passing through points of the form $(0,0,-\pi / 2+\pi n / 4), n=0,1, \ldots, 4$ and the concentration field at $\lambda=\mu=2$ 


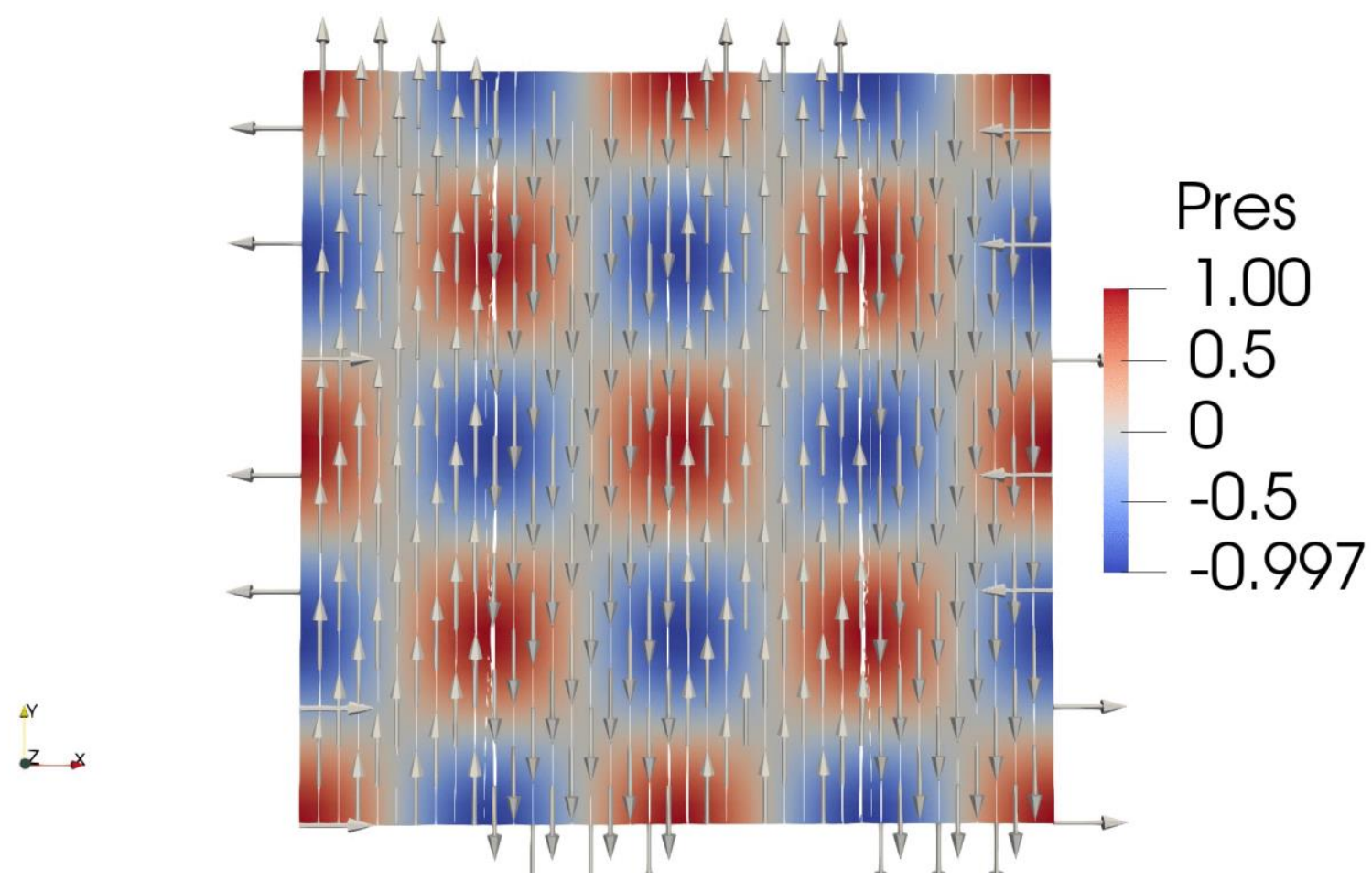

Fig. 11. The structure of flow 3 in the plane parallel to $x_{1} O x_{2}$, passing through points of the form $(0,0,-\pi / 2+\pi n / 4), n=0,1, \ldots, 4$ and the pressure field at $\lambda=\mu=2$

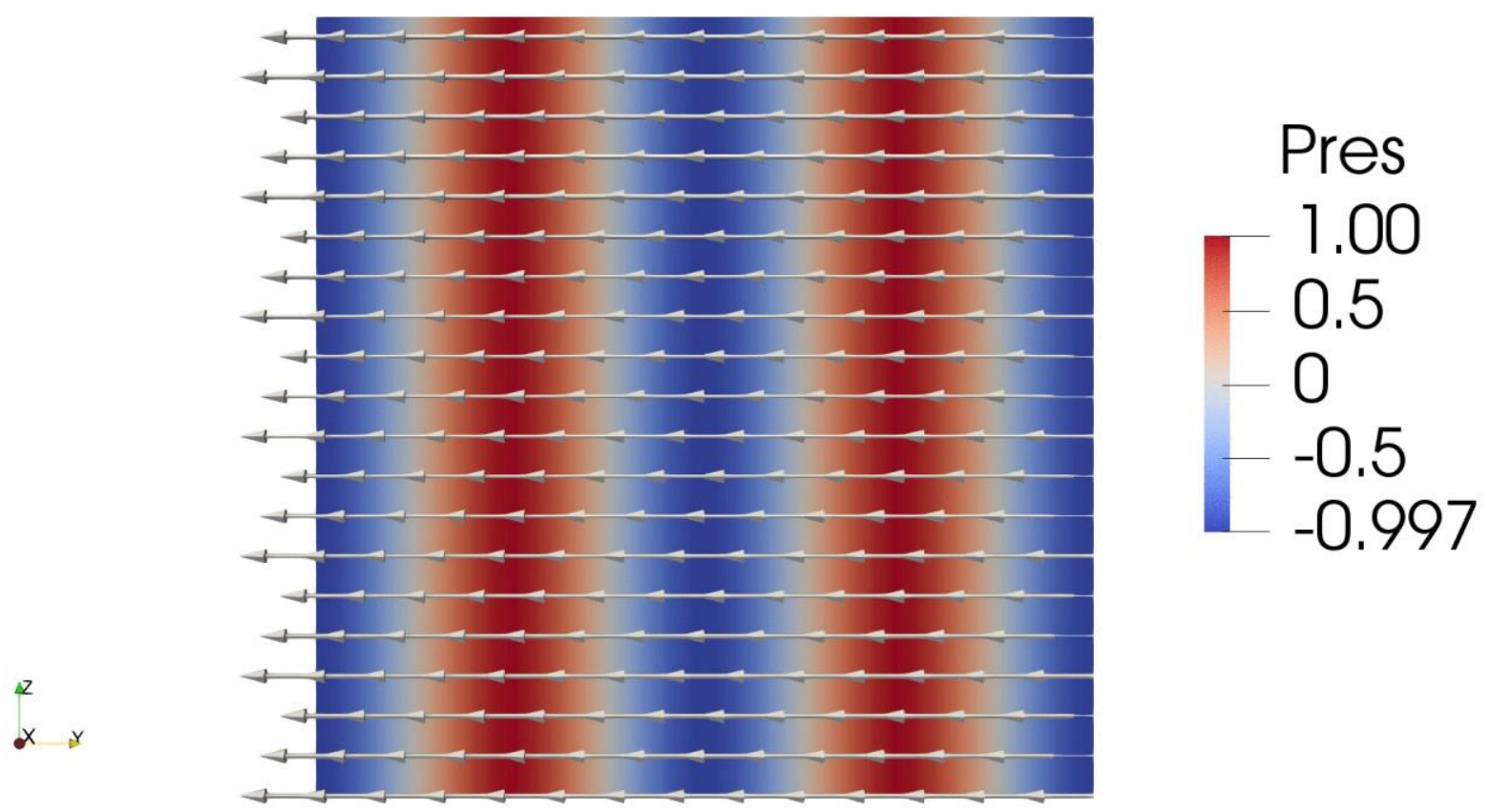

Fig. 12. The structure of flow 3 in the plane parallel to $x_{2} O x_{3}$, passing through points of the form $(-\pi / 2+\pi n / 4,0,0), n=0,1, \ldots, 4$ and the pressure field at $\lambda=\mu=2$

Now let $\lambda=2, \mu=1$. Fig. 13 illustrates the structure dependence flow from coordinate $x_{3}$ in the plane orthogonal to $O x_{3}$, in this case, $x_{3}$ sequentially takes the values $-\pi / 2,0, \pi / 2$. Fig. 13 it follows that streamlines have a structure which essentially depends on the $x_{3}$ coordinate, the impurity is located along these lines. Fig. 14 illustrates the distribution of impurities in planes perpendicular to the $O x_{2}$, these streamlines are parallel to the $O x_{1}$ axis. Distribution of impurities in planes parallel the $O x_{1}$ axis (Fig. 15) is mirror-symmetric to the distribution in Fig. 14. 


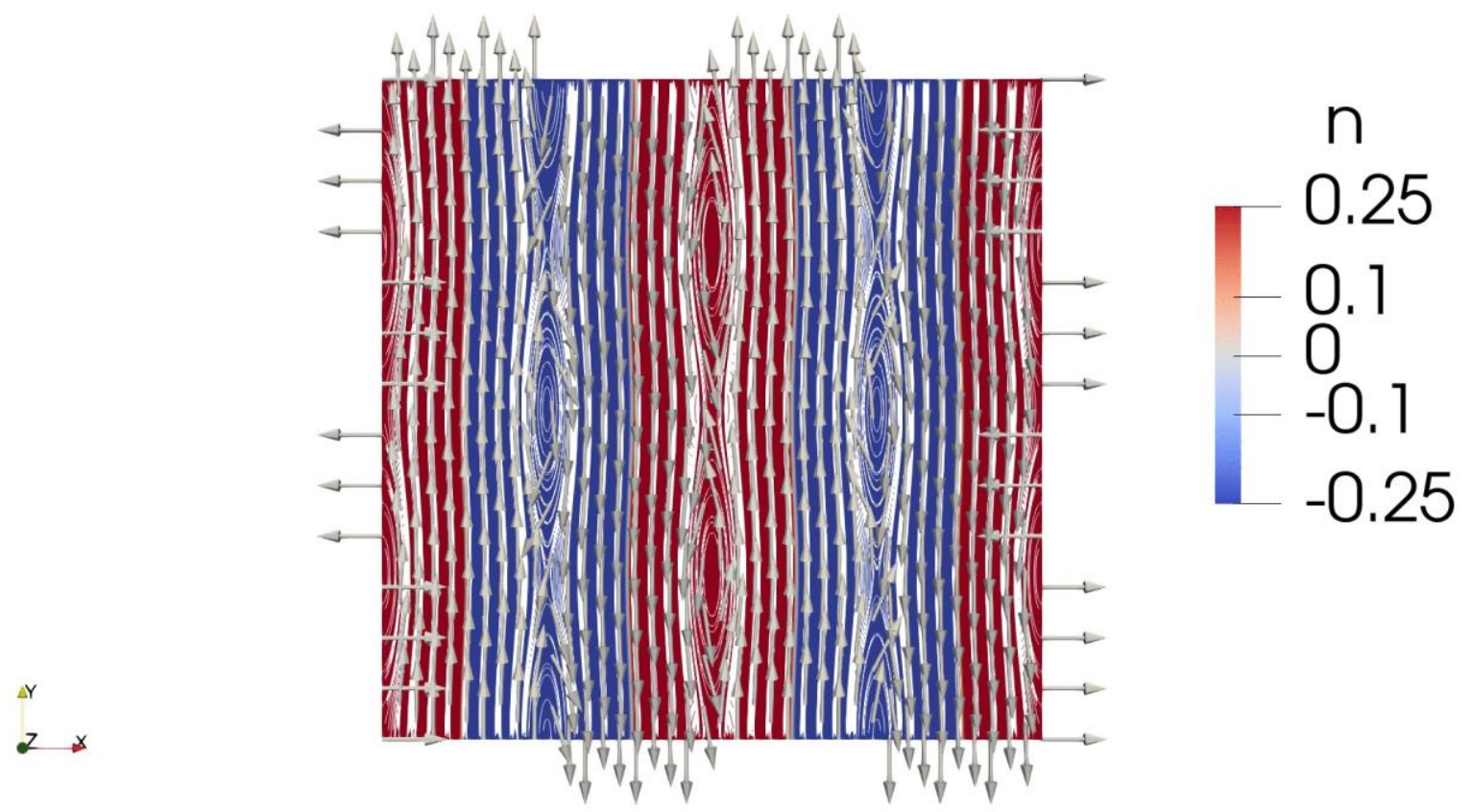

Fig. 13. The structure of flow 3 in the plane parallel to $x_{2} O x_{3}$, passing through points of the form $(0,0,-\pi / 2+\pi n / 2), n=0,1,2$ and the concentration field at $\lambda=2, \mu=1$

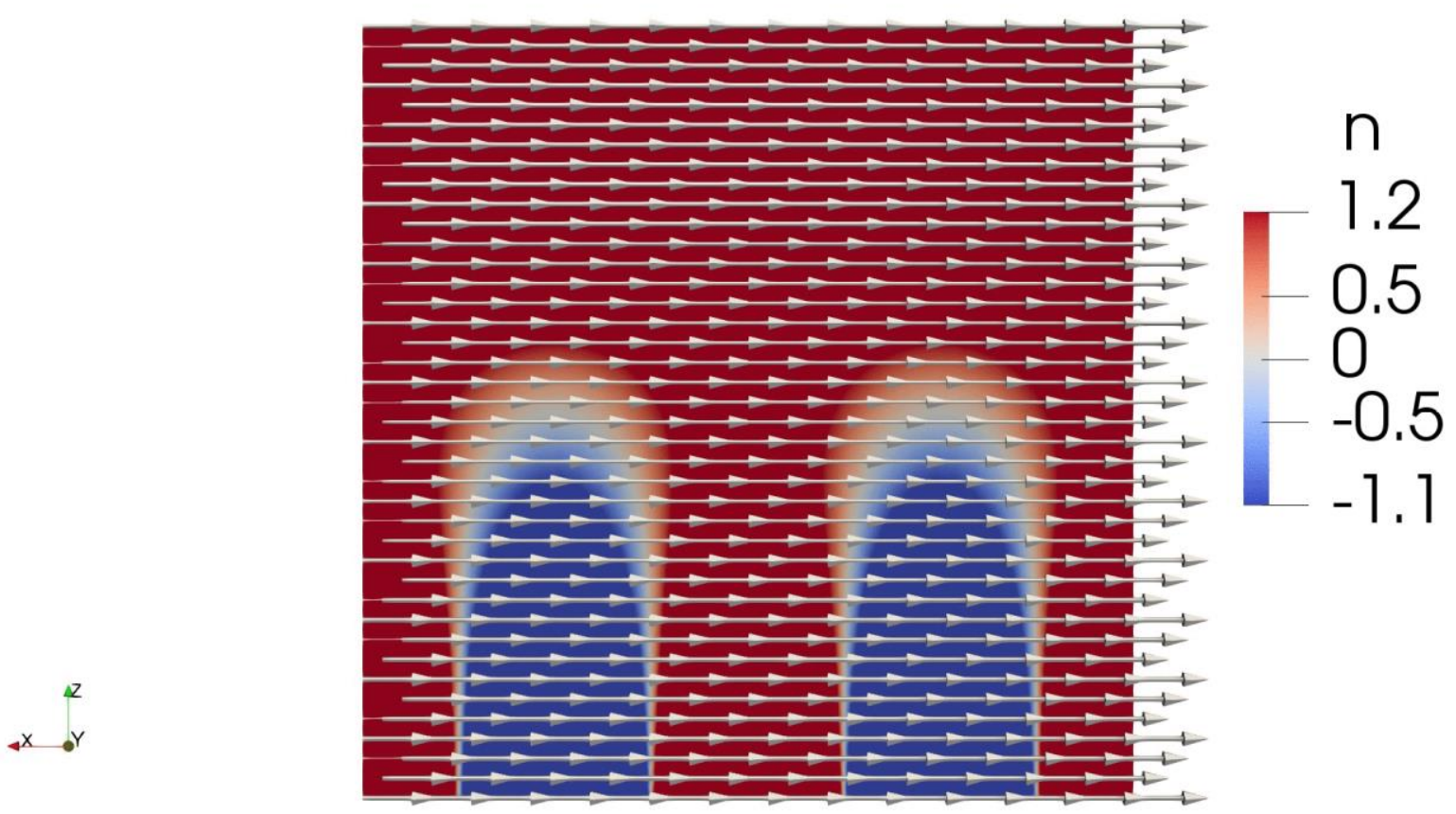

Fig. 14. The structure of flow 3 in the plane parallel to $x_{1} O x_{3}$, passing through points of the form $(0,-\pi / 2+\pi n / 2,0), n=0,1,2$ and the concentration field at $\lambda=2, \mu=1$ 


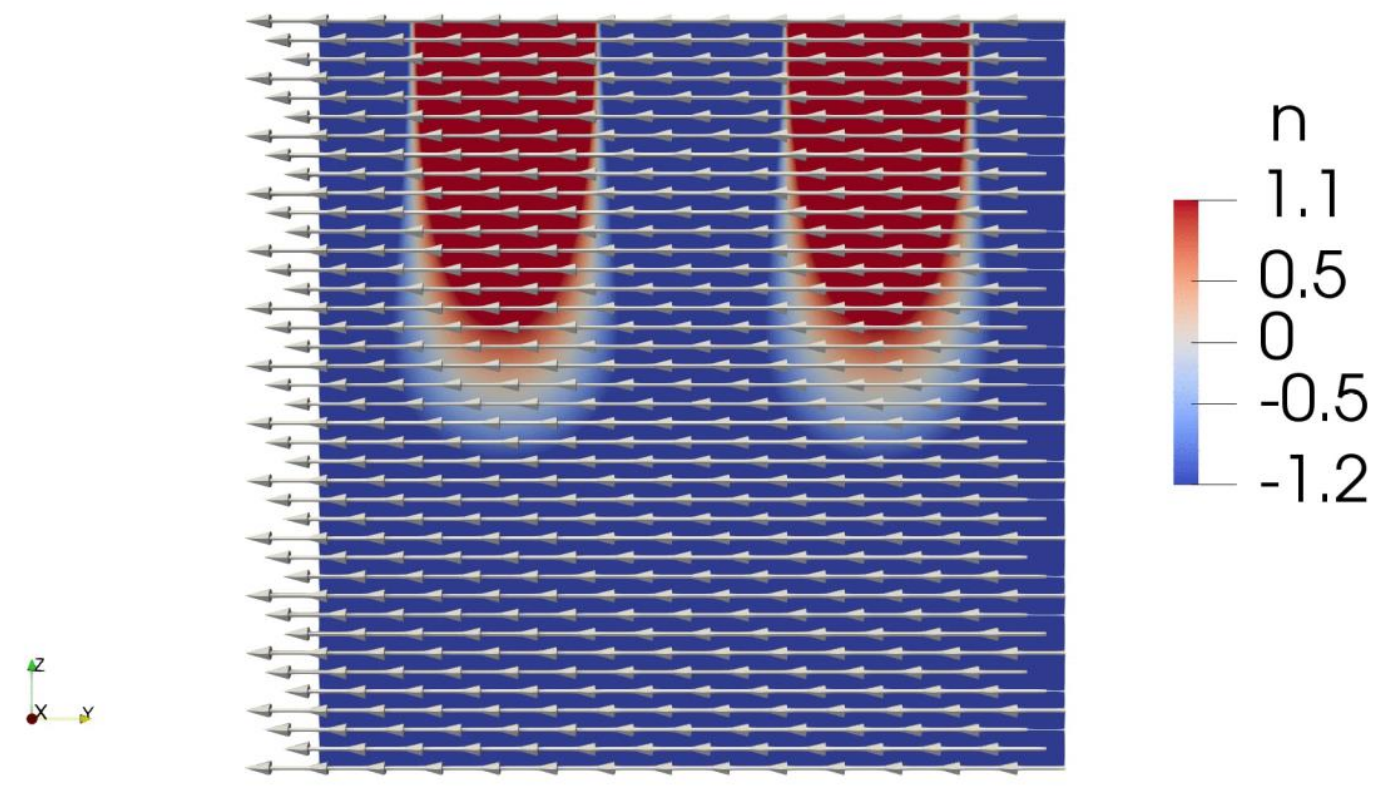

Fig. 15. The structure of flow 3 in the plane parallel to $x_{2} O x_{3}$, passing through points of the form $(-\pi / 2+\pi n / 2,0,0), n=0,1,2$ and the concentration field at $\lambda=2, \mu=1$

For the values of parameters $\lambda=1, \mu=2$ a similar flow pattern is observed as in fig. 10 with the only difference that in the presented fragment of the region the flow manages to change its direction to the opposite only once (fig. 16), and not three times as in fig. 10. Note that the structure of the flow in the plane parallel to $x_{1} O x_{2}$, passing through point with coordinates $(0,0,-\pi / 2)$ is similar to that shown in Fig. 16, however, lines are located not along the $O x_{1}$ axis, but perpendicular to them - along the $O x_{2}$ axis, the impurity is also located along streamlines. Fig. 17 illustrates the change in the flow structure in plane parallel to $x_{1} O x_{2}$ with increasing value of the coordinate $x_{3}$.

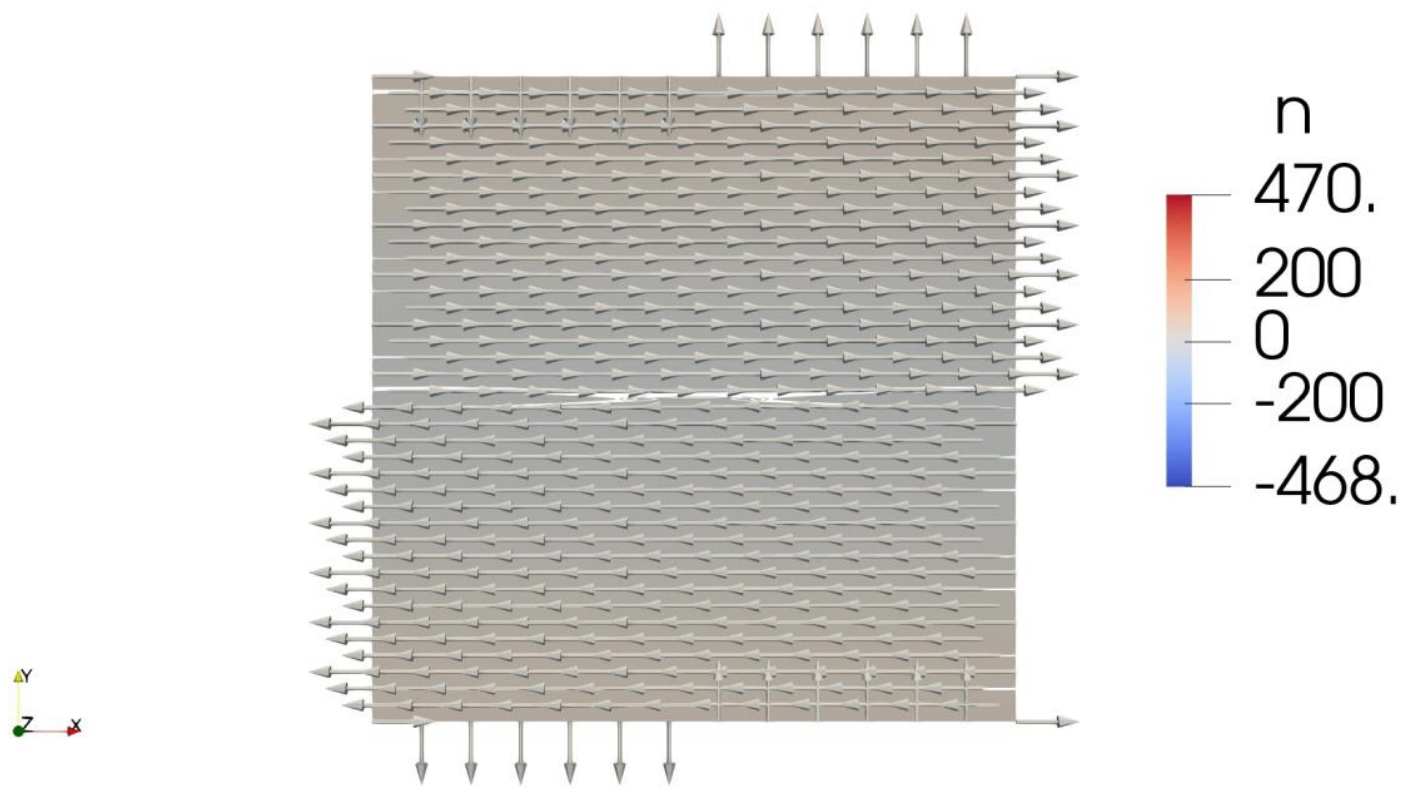

Fig. 16. Structure of flow 3 in a plane parallel to $x_{1} O x_{2}$, passing through points of the form $(0,0, \pi / 2)$ and the concentration field at $\lambda=1, \mu=2$ 


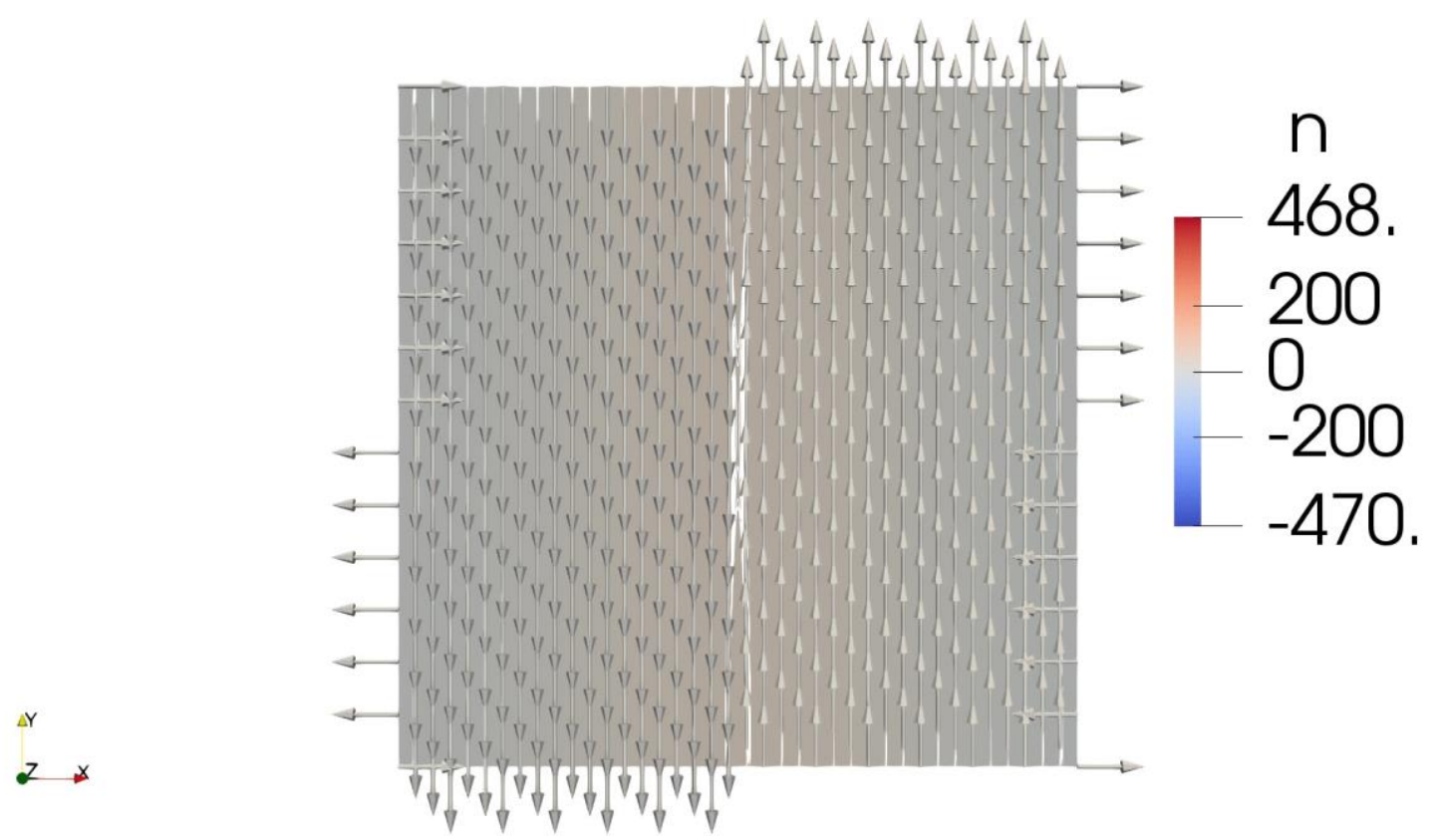

Fig. 17. The structure of flow 3 in the plane parallel to $x_{1} O x_{2}$, passing through points of the form $(0,0,-\pi / 2+\pi n / 2), n=0,1,2$ and the concentration field at $\lambda=1, \mu=2$

\section{Equations of magnetohydrodynamics}

The system of equations (1) - (4) is considered. It is assumed that on the boundary of the region flow condition of impermeability and the normal component of the velocity vector are satisfied, as well as the vector of the magnetic field strength, is equal to zero at the boundary of the region, i.e.

$$
\left.(\mathbf{u}, \mathrm{N})\right|_{\partial D}=\left.(\mathbf{H}, \mathrm{N})\right|_{\partial D}=0 \text {. }
$$

The boundary conditions in tangential directions to the boundary are specified by the narrowing of the exact solution below for this subdomain. As the flow region $D$ a sphere of radius $R$ is considered. The initial conditions are

$$
\left.\mathbf{u}\right|_{t=0}=\left\{-x_{3} \sin x_{2} ; x_{3} \sin x_{1} ; x_{1} \sin x_{2}-x_{2} \sin x_{1}\right\},\left.\mathbf{H}\right|_{t=0}=\left.2 \sqrt{\pi} \mathbf{u}\right|_{t=0} .
$$

An exact solution to problem (1) -- (4) satisfying the given initial and boundary values conditions has the form

$$
\begin{gathered}
\mathbf{u}=\exp (-\mu t)\left\{-x_{3} \sin x_{2} ; x_{3} \sin x_{1} ; x_{1} \sin x_{2}-x_{2} \sin x_{1}\right\}, \\
\mathbf{H}=2 \sqrt{\pi} \mathbf{u}, p=-\frac{\mathbf{u}^{2}}{2} .
\end{gathered}
$$

Fig. 18 illustrates the flow structure corresponding to this the exact solution, in a sphere of radius 5 over time. The figure shows that the flow on the sphere is divided into cells, which, in contrast to the previously considered flows, have different shape and size. As the radius of the sphere decreases, the cells at the poles occupy an ever larger area of the sphere, reaching almost to the equator and, accordingly, the cells located along meridians in Fig. 18 shrink and stretch along the equator. 


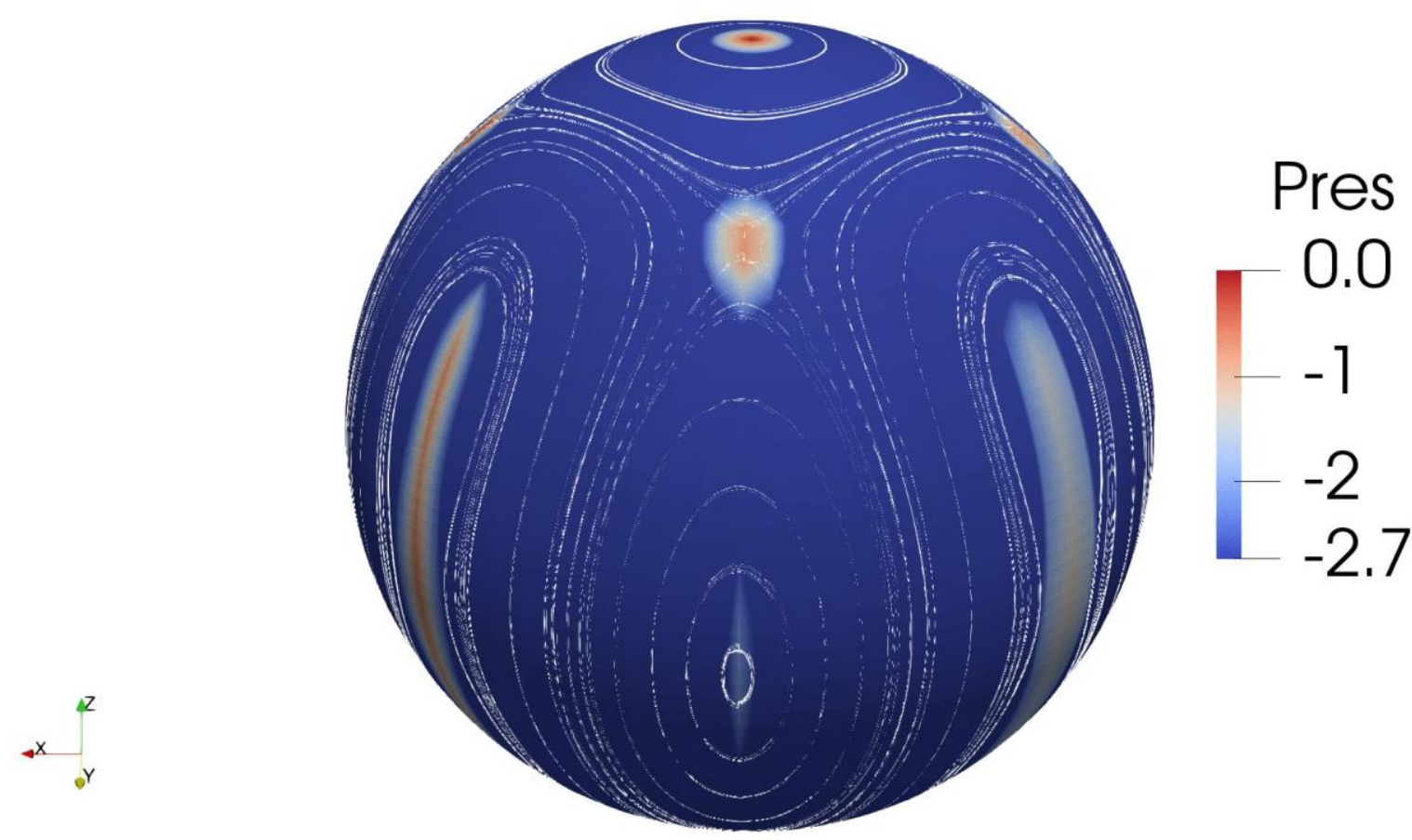

Fig. 18. Structure of flow 4 in a sphere of radius 5 and pressure field

\section{Equations of magnetohydrodynamics with impurities}

Here we consider the whole model (1) - (5), which satisfies conditions (9). As in the previous paragraph, the boundary conditions in tangential directions to the boundary are given by narrowing the exact solution given below to this subdomain. And as an area flow $D$, a sphere of radius $R$ is considered. Concentration field in all of the following solutions is assumed to be stationary, satisfying the conditions $(\mathbf{u} \cdot \nabla) n \equiv 0$ и $\Delta n \equiv 0$.

\subsection{Stationary solutions}

Several stationary solutions of equations (1) - (5) are presented below.

1. If condition (13) for the concentration field is specified on the boundary of the domain $D$, then the solution to problem (1) - (5) is

$$
\begin{gathered}
\mathbf{u}=\left\{-x_{3} x_{2} ; x_{3} x_{1} ; 0\right\}, \mathbf{H}=2 \sqrt{\pi} \mathbf{u}, p=-\frac{\mathbf{u}^{2}}{2}, \\
n=\ln \left|\frac{\sqrt{\left(\frac{x_{1}}{x_{3}}\right)^{2}+\left(\frac{x_{2}}{x_{3}}\right)^{2}+1}-1}{\sqrt{\left(\frac{x_{1}}{x_{3}}\right)^{2}+\left(\frac{x_{2}}{x_{3}}\right)^{2}+1}}\right| .1
\end{gathered}
$$

Note that if we introduce a spherical coordinate system in the domain $D$, then the latter expression for the concentration field takes the form

$$
n=\ln \left|\frac{1-|\cos \theta|}{1+|\cos \theta|}\right|,
$$

where $\theta$ is the angle between the radius vectors of a point in the area $D$ and the positive direction of the axis $O x_{3}$. Fig. 19, 20 show the streamlines in a sphere of radius 5 and pressure fields and concentration accordingly. With a change in radius, the quality of the pic- 
ture of pressure fields and concentration does not change, only the quantitative values change at each point.

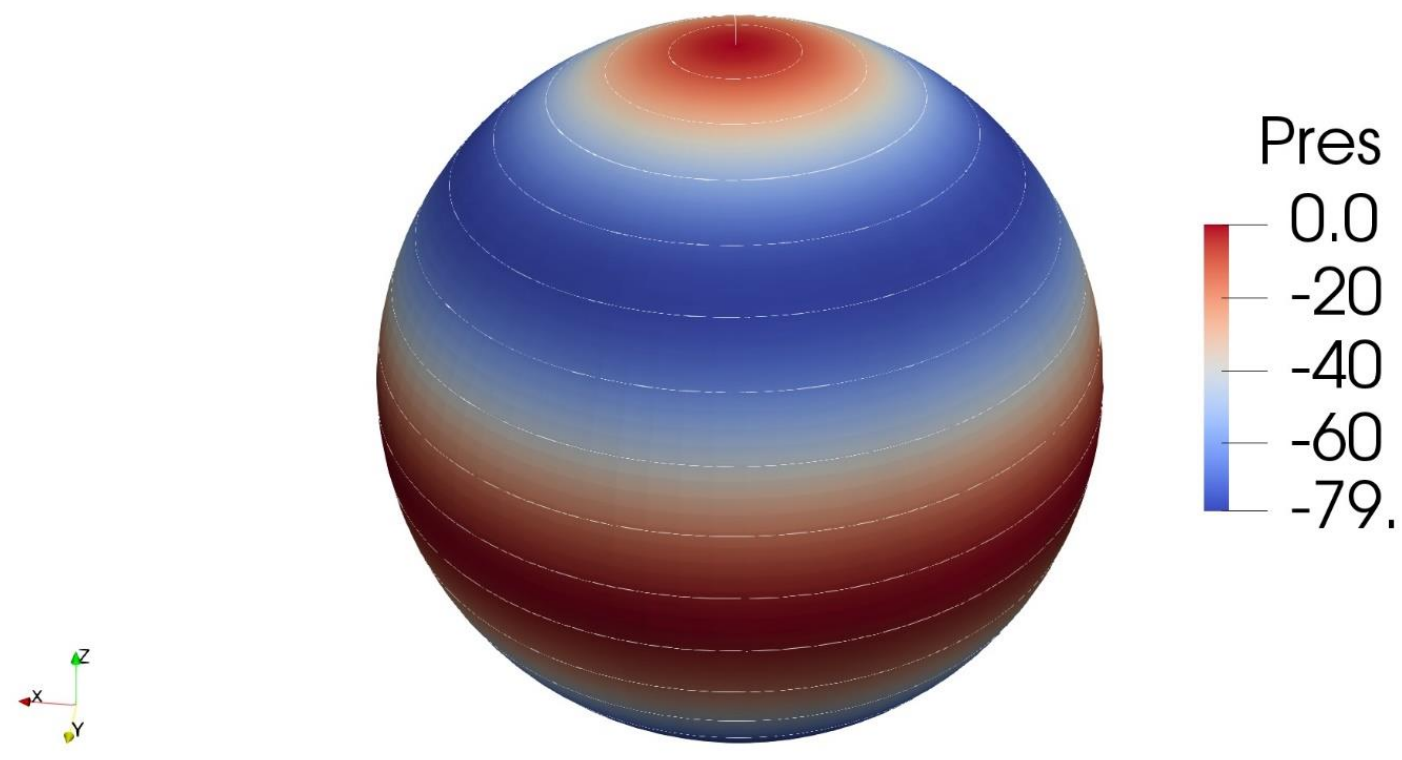

Fig. 19. 5.1.1 flow structure in a sphere of radius 5 and pressure field

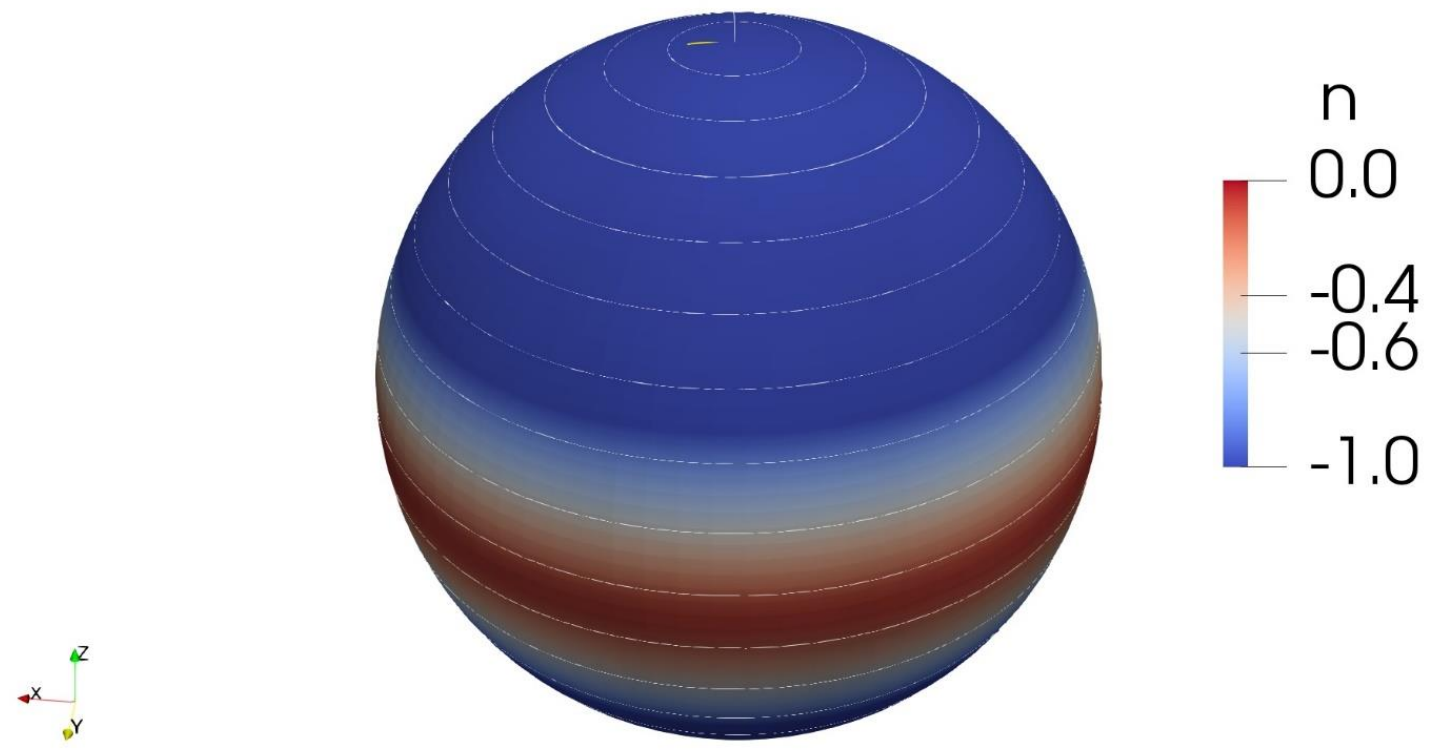

Fig. 20. 5.1.1 flow structure in a sphere of radius 5 and concentration field

2. Another stationary solution of equations (1) - (5) has the form

$$
\begin{gathered}
\mathbf{u}=\left\{x_{2}-x_{3} ; x_{3}-x_{1} ; x_{1}-x_{2}\right\}, \mathbf{H}=2 \sqrt{\pi} \mathbf{u}, p=-\frac{\mathbf{u}^{2}}{2}, \\
n=\frac{1}{\sqrt{x_{1}^{2}+x_{2}^{2}+x_{3}^{2}}} .
\end{gathered}
$$


In this case, the concentration field flux is also specified at the boundary, but here it is already nonzero. Fig. 21 shows the structure of the flow and the pressure field, corresponding to the given exact solution. Concentration field (11) on the sphere is constant.

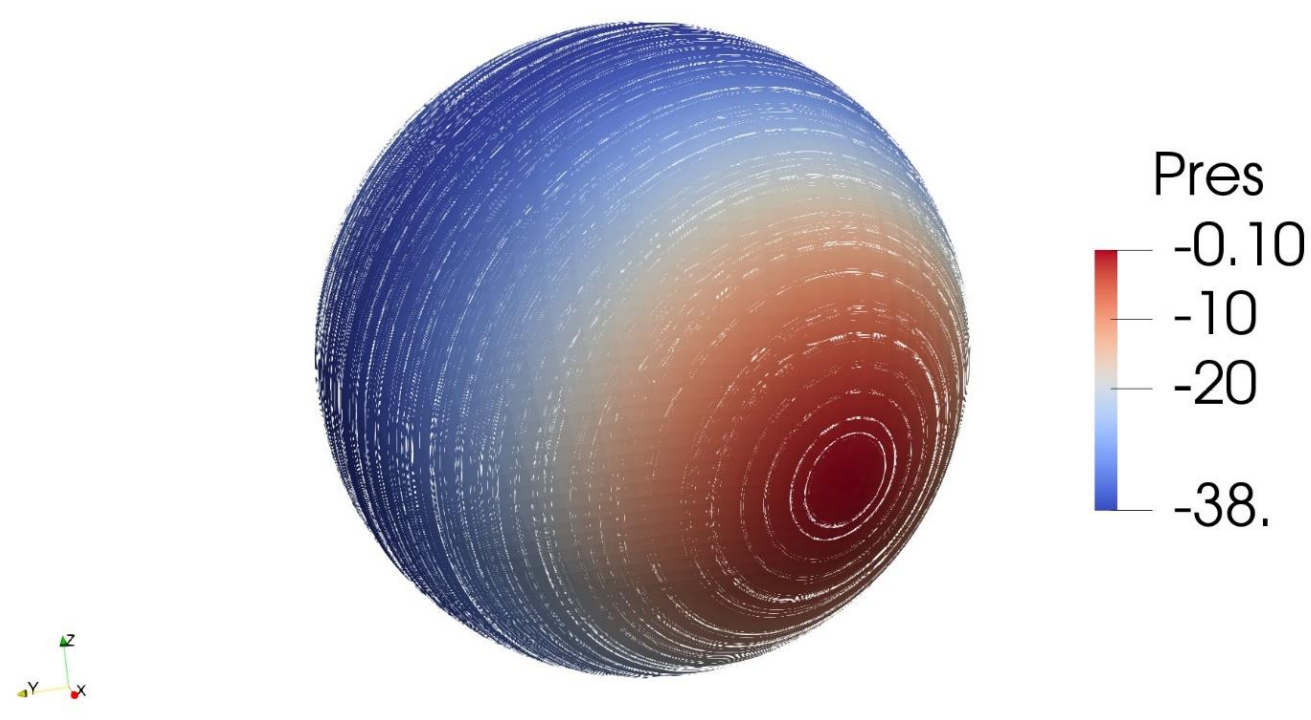

Fig. 21. Structure of flow 5.1.2 in a sphere of radius 5 and pressure field

3. The next solution to problem (1) - (5) can be obtained from the two previous ones and consists of expressions (10) and expression (11) shifted along the $O x_{3}$ axis

$$
n=\frac{1}{\sqrt{x_{1}^{2}+x_{2}^{2}+\left(x_{3}-p\right)^{2}}} .
$$

Fig. 22 illustrates the flow field parallel to the equator and the impurity flow, located along the meridians with the parameter value $p=6$, in a sphere of radius 5. Moreover, the fulfillment of the condition $(\mathbf{u} \cdot \nabla) n \equiv 0$.

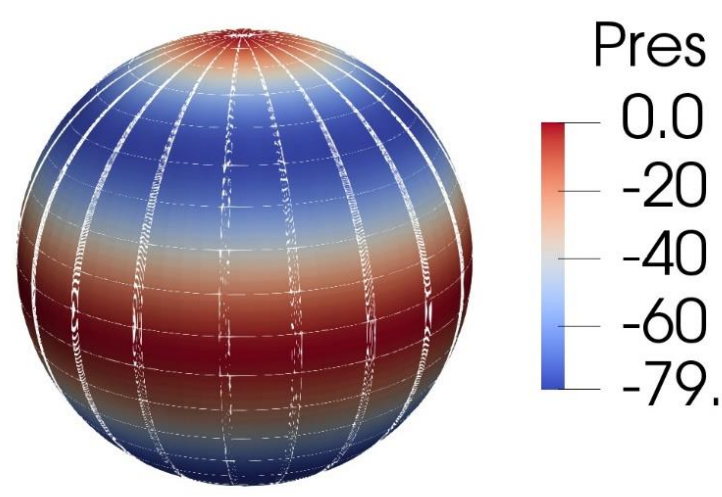

Fig. 22. Flow structure 5.1.3 (along the equator) and the impurity flow (along the meridians) in a sphere of radius 5 and field pressure 


\subsection{Non-stationary solution}

If we set the initial terms for model (1) - (5), considered in a sphere of radius $R$.

$$
\begin{gathered}
n=\frac{1}{\sqrt{x_{1}^{2}+x_{2}^{2}+x_{3}^{2}}},\left.\mathbf{H}\right|_{t=0}=\left.2 \sqrt{\pi} \mathbf{u}\right|_{t=0}, \\
\mathbf{u}=\left(\begin{array}{c}
x_{2}-3 x_{2} x_{3}-x_{3}+x_{2} \sin x_{3}-2 x_{3} \cos x_{2}+4 x_{2} \sin \sqrt{2} x_{3}-x_{3} \sin \sqrt{2} x_{2} \\
-x_{1}+3 x_{1} x_{3}+x_{3}+-x_{1} \sin x_{3}+3 x_{3} \sin x_{1}+x_{3} \cos \sqrt{2} x_{1}-4 x_{1} \sin \sqrt{2} x_{3} \\
x_{1}-x_{2}+2 x_{1} \cos x_{2}-3 x_{2} \sin x_{1}+x_{1} \sin \sqrt{2} x_{2}-x_{2} \cos \sqrt{2} x_{1}
\end{array}\right),
\end{gathered}
$$

then the solution to problem (1) - (5) satisfying conditions (9) has the form

$$
\begin{gathered}
n=\frac{1}{\sqrt{x_{1}^{2}+x_{2}^{2}+x_{3}^{2}}}, \mathbf{H}=2 \sqrt{\pi} \mathbf{u}, p=-\frac{\mathbf{u}^{2}}{2}, \\
\mathbf{u}=\left(\begin{array}{c}
x_{2}-3 x_{2} x_{3}-x_{3} \\
-x_{1}+3 x_{1} x_{3}+x_{3} \\
x_{1}-x_{2}
\end{array}\right)+e^{-\mu t}\left(\begin{array}{c}
x_{2} \sin x_{3}-2 x_{3} \cos x_{2} \\
-x_{1} \sin x_{3}+3 x_{3} \sin x_{1} \\
2 x_{1} \cos x_{2}-3 x_{2} \sin x_{1}
\end{array}\right)+e^{-2 \mu t}\left(\begin{array}{c}
4 x_{2} \sin \sqrt{2} x_{3}-x_{3} \sin \sqrt{2} x_{2} \\
x_{3} \cos \sqrt{2} x_{1}-4 x_{1} \sin \sqrt{2} x_{3} \\
x_{1} \sin \sqrt{2} x_{2}-x_{2} \cos \sqrt{2} x_{1}
\end{array}\right) .
\end{gathered}
$$

Fig. 23 illustrates the time variation of streamlines and pressure fields for the exactly considered solution of the MHD equations. Streamlines near poles located in parallels, while streamlines closer to the equator are located along areas that change markedly over time.

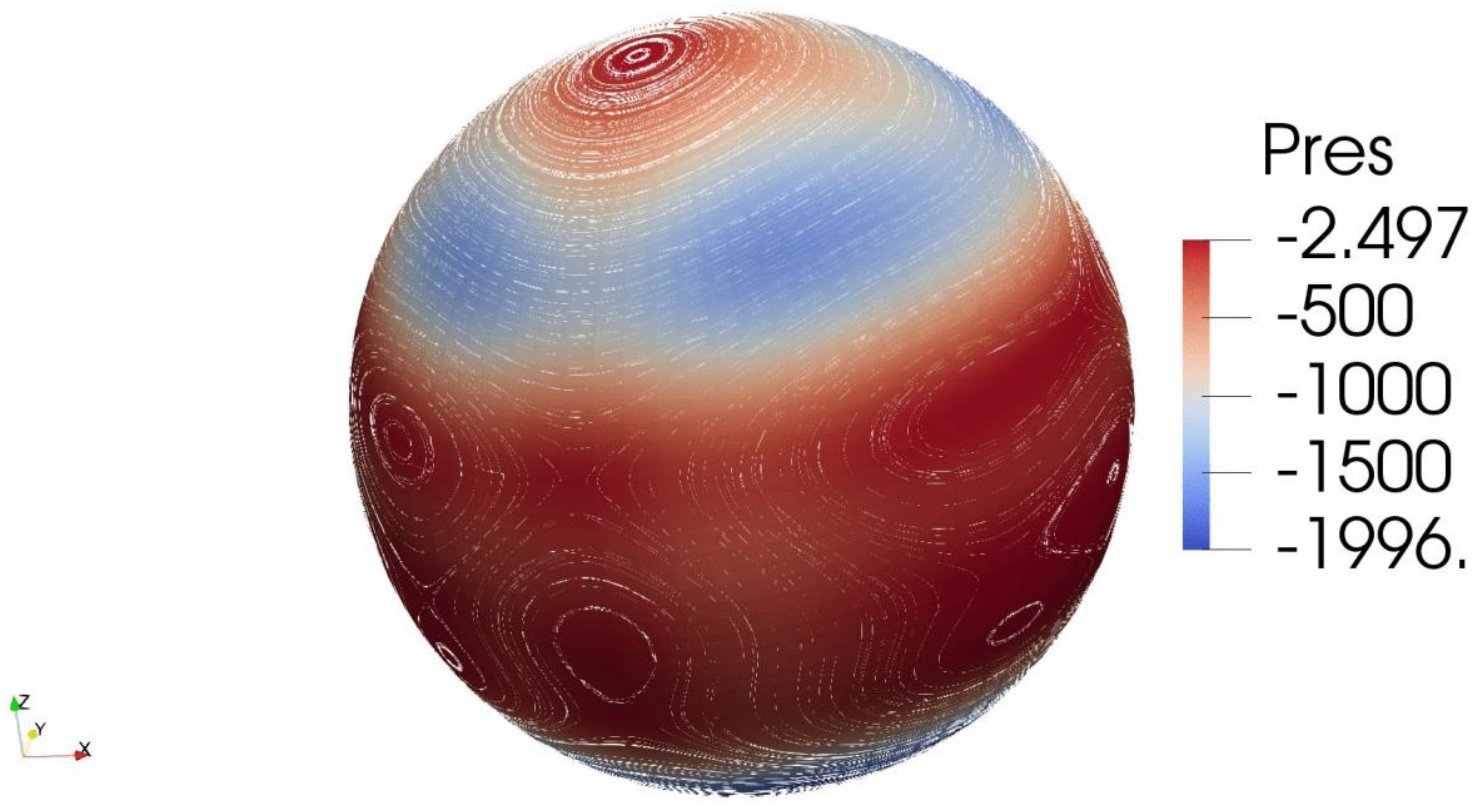

Fig. 23. Structure of a 5.2 flow in a sphere of radius 5 and a pressure field

Note that condition (9) is satisfied for a sphere of arbitrary radius. Thus, the considered fluid flows in paragraphs 4,5 are stratified into layers - spheres of fixed radius. The solutions presented in paragraph 5.1 points 1 and 2, are special cases of solution 5.3 and correspond to the linear and nonlinear parts the first term of the velocity vector $\mathbf{u}$. 


\section{Conclusion}

Exact solutions of the MHD equations, supplemented by the convection-diffusion equation, describing the flow of a viscous conducting incompressible fluid, taking into account the presence of impurities in the flow field, are considered in this work. The exact solutions found can be used to verify the results of modeling such flows. In addition, the paper presents the classes of flows corresponding to the flow of liquid in a porous medium. Understanding and analyzing the structure of such complex flows is not possible without their visual presentation. The task of visualizing the flow field can be handled by various currently available software tools. ParaView (https://www.paraview.org) was used when writing this article.

This work was supported by the Russian Foundation for Basic Research, projects No. 1847-860005, 18-47-860004.

\section{References}

1. Landau L.D. Lifshits E. M. Teoreticheskaya fizika: Uchebn. posob.: Dlya vuzov. V 10 t. T. VIII. Elektrodinamika sploshnykh sred [Theoretical physics: Textbook. manual: For universities. In 10 volumes, V. VIII. Continuous media electrodynamics.] - 4th ed., Stereo.Moscow: FIZMATLIT. 2005. 656 p. [in Russian]

2. Kulikovsky A.G., Lyubimov G.A. Magnitnaya gidrodinamika [Magnetic hydrodynamics]. - Ed. 2nd. rev. and add. - Moscow: Logos. 2005.-328 p. [in Russian]

3. Betelin V.B., Galkin V.A. Control of Incompressible Fluid Parameters in the case of Time_Varying Flow Geometry // Doklady Mathematics. 2015. V. 92. No 1. p. 511-513.

4. V. A. Galkin, A. O. Dubovik, A. A. Epifanov. Approximate Methods for Equations of incompressible fluid // Computational Mathematics and Mathematical Physics. 2017, V. 57, no. 2, p. 272-280.

5. Volpin S.G. Yudin V.A., Kats R.M., Afanaskin I.V., Galkin V.A. Primeneniye superkomp'yuternykh tekhnologiy- klyuch k resheniyu problem povysheniya nefteotdachi na mestorozhdeniyakh Rossii [Application supercomputer technologies are the key to solving the problems of enhanced oil recovery at deposits of Russia] // In collection: $\mathrm{SPb}$ scientific. forum. VIII meeting of Nobel laureates awards. Sankt-Petersburg. 2013.S. 90-92. [in Russian]

6. Galich N.E. Teplovaya neustoychivost' i proboy dvizhushchikhsya vyazkikh zhidkostey v elektricheskom pole i pri pogloshchenii sveta [Thermal instability and breakdown of moving viscous fluids in electric field and absorption of light] // Zhurnal tekhnicheskoy fiziki. 1989. vol. 59. no. 7. p. 10-17. [in Russian]

7. Altoiz B.A., Savin N.V., Shatagina E.A. Vliyaniye teplovydeleniya v mikroprosloyke zhidkosti pri izmerenii yeye vyazkosti [Influence of heat dissipation in micro-layer liquid when measuring its viscosity] // Zhurnal tekhnicheskoy fiziki. 2014.Vol. 84.V. 5.P. 2127. [in Russian]

8. Kushtanova G.G. Fizika geosfery [Physics of the geosphere]. Kazan. From Kazan State University. 2004. 44 p. [in Russian]

9. Semenov N.N. Tsepnyye reaktsii [Chain reactions]. Moscow: Gomkhimtekhizdat. 1934. [in Russian]

10. Shestakov V.M. Hydrogeodynamica [Hydrogeodynamics]. Moscow: Moscow State University. 1995. 368 p. [in Russian]

11. Sukhinov A.I., Chistyakov A.E., Protsenko E.A. Raznostnaya skhema dlya resheniya zadach gidrodinamiki pir bol'shikh setochnykh chislakh Pekle [Difference scheme for solving problems hydrodynamics of large grid Peclet numbers] // Komp'yuternyye issledovaniya i modelirovaniye. 2019. V. 11.No. 5. p. 833-848. [in Russian]

12. Semenyakina A.A. Parallel'noye resheniye zadach diffuzii-konvektsii na osnove skhem povyshennogo poryadka tochnosti [Parallel solution of diffusion-convection problems based on schemes increased order of accuracy] // In collection Superkomp'yuternyye 
tekhnologii (SKT-2016). Materials of the 4th All-Russian Scientific and Technical Conference. In 2 volumes. 2016.S. 281-285. [in Russian]

13. Kleschenkov A.V., Sorokina V.V., Chikin A.L., Chikina L.G. Chislennoye modelirovaniye protsessa raspredeleniya rechnykh nanosov dona $v$ taganrogskom zalive s pomoshch'yu matematicheskoy modeli [Numerical simulation the process of distribution of river sediments of the Don in the Taganrog Bay using mathematical model] // Ekologiya. Ekonomika. Informatika. Seriya: sistemnyy analiz i modelirovaniye ekonomicheskikh i ekologicheskikh sistem. 2019. V. 1.No. 4.P. 217-222. [in Russian]

14. Sukhinov A.I., Chistyakov A.E., Nikitina A.V., Chistyakova A.E. Matematicheskoye modelirovaniye gidrodinamiki protsessov perenosa soley $\mathrm{i}$ tepla $\mathrm{v}$ melkovodnykh vodoyemakh [Mathematical modeling the hydrodynamics of salt and heat transfer processes in shallow water reservoirs] // Collection of All-Russian Scientific Conf. from int. participation of "Zemlya i kosmos" to centenary of Academician of RAS K. Ya. Kondratyev. Digest of articles. 2020. p. 307-311. [in Russian]

15. Betelin V.B., Galkin V.A., Dubovik A.O. Exact Solutions of Incompressible NavierStokes Equations in the Case of Oil and Gas Industrial Problems // Doklady Mathematics. 2020.Vol. 102. No 3. p. 456-459.

16. Betelin V.B., Galkin V.A., Gorelikov A.V. Predictor-Corrector Algorithm for the Numerical Solution of the Magnetic Field Equation in Viscous Incompressible MHD Problems // Doklady Mathematics. 2015. V. 92. No. 2. p. 618-621. 